\title{
Relativistic phase shifts for Dirac particles interacting with weak gravitational fields in matter-wave interferometers*
}

\author{
Christian J. Bordé1,2,3 and Jean-Claude Houard ${ }^{1}$, and Alain Karasiewicz ${ }^{1}$ \\ ${ }^{1}$ Laboratoire de Gravitation et Cosmologie Relativistes, Université Pierre et Marie Curie, \\ 4 Place Jussieu, 75252 Cedex 05, Paris, France \\ ${ }^{2}$ Laboratoire de Physique des Lasers, UMR 7538 CNRS, Université Paris-Nord, \\ Avenue J.-B. Clément, 93430 Villetaneuse, France \\ ${ }^{3}$ Institut für Quantenoptik, Universität Hannover, Welfengarten 1, \\ 30167 Hannover, Germany
}

November 23, 2018

\begin{abstract}
We present a second-quantized field theory of massive spin one-half particles or antiparticles in the presence of a weak gravitational field treated as a spin two external field in a flat Minkowski background. We solve the difficulties which arise from the derivative coupling and we are able to introduce an interaction picture. We derive expressions for the scattering amplitude and for the outgoing spinor to first-order. In several appendices, the link with the canonical approach in General Relativity is established and a generalized stationary phase method is used to calculate the outgoing spinor. We show how our expressions can be used to calculate and discuss phase shifts in the context of matter-wave interferometry (especially atom or antiatom interferometry). In this way, many effects are introduced in a unified relativistic framework, including spingravitation terms: gravitational red shift, Thomas precession, Sagnac effect, spin-rotation effect, orbital and spin Lense-Thirring effects, de Sitter geodetic precession and finally the effect of gravitational waves. A new analogy with the electromagnetic interaction is pointed out.
\end{abstract}

\section{Introduction}

The development of high accuracy atom interferometers, used as clocks in the microwave or in the optical domain, as inertial sensors (gyros, gravimeters, gradiometers...) or for the determination of atomic masses and of the fine structure constant [1, 2, 3, , 4, 5, 6, 7, 8, 9, 10, 11], requires now a framework to describe the interference of atom waves in a rigorous way. On one hand, one needs to investigate general relativistic effects including those involving the spin of the atoms and, on the other hand, it is necessary to take into account the statistical properties (bosonic or fermionic) of the interfering particles, given the development of coherent atom wave sources and also for a proper treatment of the detection noise. One would also like to be able to discuss the propagation of antimatter in interferometers in the presence of gravitation and as suggested in reference [22] the properties of coherent antimatter waves (generated by an antiatom laser such as an antihydrogen Bose-Einstein condensate). This is possible only within relativistic quantum field theory. Atoms (or antiatoms), in a given eigenstate of the internal atomic Hamiltonian, are considered as elementary particles having a rest mass fixed by the energy of the atomic level and a spin equal to the total angular momentum of the atom in that level. In this paper, we shall consider only Dirac particles for illustration. This is the simplest example of particles with spin which still contains most interesting effects related to spin and applies to neutron or electron interferometry as

\footnotetext{
*to appear in: C. Lämmerzahl, C.W.F. Everitt, F.W. Hehl (eds.): Gyros, Clocks, and Interferometers: Testing Relativistic Gravity in Space, Springer-Verlag, Berlin 2000
} 
a special case. The generalization to others spin values is possible along similar lines with Dirac-type equations (Bargmann-Wigner [27], de Broglie fusion method [28], Durand [29]). Our overall goal is to introduce gravitation and general relativistic effects at the quantum level of modern atomic physics and quantum optics experiments. For this, we propose an extension of our first paper on atom interferometry in General Relativity [12], which includes now a second-quantization scheme for the atom waves in the presence of gravitational and electromagnetic fields. The point of view adopted in this paper is the extrinsic point of view using purely quantum field theory in a flat Minkowski background. The connection with the canonical intrinsic approach, using Dirac equation in curved space-time, is made in Appendices A and B. The reader who wishes to start with this canonical approach is thus invited to read first these appendices.

In the main text, we begin right away with the minimal coupling Lagrangian in flat space-time, which is identical to the one derived in curved space-time for a standard choice of tetrads. Then, we proceed with the quantization of the Dirac field and we emphasize the difficulties which arise because of the derivative coupling. These difficulties are solved in a consistent scheme which allows also to define an interaction picture. The evolution operator and the $S$-matrix are constructed and we demonstrate explicitly a conjecture of Gupta. These results are used to derive formulas for the transition amplitude and for the outgoing spinor in the weak-field approximation, first in configuration space and second in the momentum representation. An expansion in the perturbation wave vector $(\hbar k / m c)$ is used to retrieve various physical effects, some of which are well-known. A new analogy with the electromagnetic interaction is presented which includes all components of the field $h^{\mu \nu}$ and generalizes gravitoelectric and gravitomagnetic interactions. In Appendices C and D, calculations of the outgoing spinor are sketched, first with a generalized stationary phase method in configuration space and second in the momentum representation.

\section{Lagrangian theory}

Considered as a field theory in flat spacetime, the theory describing the interaction of matter with a given gravitational field will be defined by a Lagrangian density of the following form 15, 19]:

$$
\mathcal{L}=\mathcal{L}_{0}-\frac{1}{2} h^{\mu \nu} T_{\mu \nu}
$$

where $h^{\mu \nu}$ is the given external field, and where $\mathcal{L}_{0}$ is the free Lagrangian density of the matter field and $T_{\mu \nu}$ the corresponding stress-energy tensor. For a Dirac field, one has respectively円, in symmetrical form,

$$
\begin{aligned}
\mathcal{L}_{0} & =\frac{\hbar c}{2}\left[\bar{\Psi}\left(i \gamma^{\mu} \overrightarrow{\partial_{\mu}}-\frac{m c}{\hbar}\right) \Psi+\bar{\Psi}\left(-i \gamma^{\mu} \overleftarrow{\partial_{\mu}}-\frac{m c}{\hbar}\right) \Psi\right] \\
T_{\mu \nu} & =-\eta_{\mu \nu} \mathcal{L}_{0}+\frac{\hbar c}{4}\left[\bar{\Psi}\left(i \gamma_{\mu} \overrightarrow{\partial_{\nu}}-i \overleftarrow{\partial_{\nu}} \gamma_{\mu}\right) \Psi+\bar{\Psi}\left(i \gamma_{\nu} \overrightarrow{\partial_{\mu}}-i \overleftarrow{\partial_{\mu}} \gamma_{\nu}\right) \Psi\right]
\end{aligned}
$$

The total Lagrangian density (11) then becomes

$$
\mathcal{L}=\left(1+\frac{1}{2} h\right) \mathcal{L}_{0}-\frac{1}{4} i \hbar c h^{\mu \nu} \bar{\Psi}\left(\gamma_{\mu} \vec{\partial}_{\nu}-\overleftarrow{\partial}_{\nu} \gamma_{\mu}\right) \Psi
$$

wheref $h=h^{\mu}{ }_{\mu}=\eta_{\mu \nu} h^{\mu \nu}$. This Lagrangian density can also be considered as obtained from the Lagrangian density valid in General Relativity for the interaction of the Dirac field with a prescribed gravitational field in the linear approximation (see the Appendices).

\footnotetext{
${ }^{1}$ The conventions used here for the metric and for the Dirac equation and matrices are generally those of [17]. In particular, the signature of the metric is taken as $(+,-,-,-)$. Greek indices $\mu, \nu, \ldots$ run from 0 to 3 and latin indices run from 1 to 3 . The space-time 4 -vector is written $x=\left(x^{0}, \vec{x}\right)=(c t, \vec{x})$. The partial derivatives with the right arrow act on the right and those with the left arrow on the left.

${ }^{2}$ The term $(h / 2) \mathcal{L}_{0}$ in (4) comes from the term containing $\mathcal{L}_{0}$ in (3), and was omitted in [16]. However, $\mathcal{L}_{0}$ which vanishes when the free Dirac equation is satisfied, does not vanish here.
} 
The equations for $\Psi$ and $\bar{\Psi}$ derived from (4) are the followings]:

$$
\begin{gathered}
{\left[\left(1+\frac{1}{2} h\right)\left(i \gamma^{\mu} \vec{\partial}_{\mu}-m c / \hbar\right)-\frac{i}{2} h^{\mu \nu} \gamma_{\mu} \vec{\partial}_{\nu}-\frac{i}{4} \partial_{\nu} h^{\mu \nu} \gamma_{\mu}+\frac{i}{4} \partial_{\mu} h \gamma^{\mu}\right] \Psi=0} \\
\bar{\Psi}\left[\left(-i \gamma^{\mu} \overleftarrow{\partial}_{\mu}-m c / \hbar\right)\left(1+\frac{1}{2} h\right)+\frac{i}{2} \overleftarrow{\partial}_{\nu} \gamma_{\mu} h^{\mu \nu}+\frac{i}{4} \partial_{\nu} h^{\mu \nu} \gamma_{\mu}-\frac{i}{4} \partial_{\mu} h \gamma^{\mu}\right]=0
\end{gathered}
$$

They admit the conserved current

$$
j^{\mu}=c \bar{\Psi}\left[\gamma^{\mu}+\frac{1}{2} h \gamma^{\mu}-\frac{1}{2} h^{\mu \nu} \gamma_{\nu}\right] \Psi,
$$

which coincides with the usual Dirac current when the gravitational field vanishes.

In order to stress some other differences and analogies with electromagnetism, we may write the equation for $\Psi$ with a covariant derivative in the usual sense of non-Abelian gauge field theories in flat space-time

$$
i \gamma^{\nu}\left(\vec{\partial}_{\nu}+\frac{i}{4} \sigma^{\lambda \mu} \partial_{\lambda} h_{\mu \nu}-\frac{1}{2} h_{\nu}{ }^{\alpha} \vec{\partial}_{\alpha}\right) \Psi-\frac{m c}{\hbar} \Psi=0
$$

where

$$
\sigma^{\mu \nu}=\frac{i}{2}\left(\gamma^{\mu} \gamma^{\nu}-\gamma^{\nu} \gamma^{\mu}\right)
$$

and where the factor $\left(1+\frac{1}{2} h\right)$ has been removed. The Poincaré generators are associated with gauge fields and a local gauge invariancef

$$
\begin{aligned}
\Psi^{\prime}(x) & =\left[1+\frac{i}{8} \sigma^{\lambda \mu}\left(\partial_{\lambda} \xi_{\mu}-\partial_{\mu} \xi_{\lambda}\right)-\xi^{\lambda} \partial_{\lambda}\right] \Psi(x) \\
h_{\mu \nu}^{\prime}(x) & =h_{\mu \nu}(x)-\partial_{\mu} \xi_{\nu}-\partial_{\nu} \xi_{\mu}
\end{aligned}
$$

\section{Quantization}

We want now to proceed with the quantization of the field $\Psi$ submitted to the interaction defined by (目). When they are applied directly, the standard methods lead to some difficulties coming from the presence of a derivative coupling in the Lagrangian. These problems are, first, briefly discussed, then, a solution is presented allowing the quantization together with the definition of the interaction picture.

\subsection{Difficulties with the derivative coupling}

Two methods can be used, a priori, according to whether the Lagrangian is taken under a symmetrical or a asymmetrical form.

\subsubsection{Symmetrical Lagrangian.}

Starting from the symmetrical Lagrangian (雨), the usual anticommutation relations will be obtained from the following expression of the conjugate momentum

$$
\Pi=2 \frac{\partial \mathcal{L}}{\partial \dot{\Psi}}, \quad \text { with } \quad \dot{\Psi}=\partial_{t} \Psi
$$

\footnotetext{
${ }^{3}$ If one considers these equations as first-order equations with respect to the $h^{\mu \nu}$ 's, the factor $(1+h / 2)$ in the first term can be replaced by 1 . But it can be shown that the equations so obtained cannot be derived from a Lagrangian, if this latter is restricted to depend linearly on $\Psi$ and $\bar{\Psi}$, and to admit first-order derivatives only.

${ }^{4}$ Differences with usual Yang-Mills theories come from the non-commutation of the Lorentz generators with the Dirac matrices and from the fact that the translation generators act on space-time itself.
} 
which gives

$$
\Pi=i \hbar \bar{\Psi}\left[\left(1+\frac{1}{2} h\right) \gamma^{0}-\frac{1}{2} h^{0 \mu} \gamma_{\mu}\right]
$$

and

$$
\left\{\Psi_{\alpha}(x), \Pi_{\beta}(y)\right\}_{x^{0}=y^{0}}=i \hbar \delta_{\alpha \beta} \delta(\vec{x}-\vec{y})
$$

where $\alpha$ and $\beta$ are spinorial indices.

By introducing the matrix (depending on the coordinates)

$$
\gamma^{-1}=\left(1+\frac{1}{2} h\right) \gamma^{0}-\frac{1}{2} h^{0 \mu} \gamma_{\mu},
$$

these formulas can be rewritten

$$
\Pi=i \hbar \bar{\Psi} \gamma^{-1},
$$

and

$$
\left\{\Psi_{\alpha}(x), \bar{\Psi}_{\beta}(y)\right\}_{x^{0}=y^{0}}=(\gamma(x))_{\alpha \beta} \delta(\vec{x}-\vec{y}) .
$$

For the free field the matrix $\gamma(x)$ reduces to $\gamma^{0}$. However, it appears that the Heisenberg equations, which are generally equivalent to the equations of motion, are not satisfied here under their most usual forms. To write the formulas in a condensed form, let us introduce the two operators

$$
\begin{aligned}
& \overrightarrow{\mathcal{D}}=\hbar c\left[\left(1+\frac{1}{2} h\right)\left(-i \gamma^{k} \overrightarrow{\partial_{k}}+m c / \hbar\right)+\frac{i}{2} h^{\mu k} \gamma_{\mu} \overrightarrow{\partial_{k}}+\frac{i}{4}\left(\partial_{\nu} h^{\mu \nu}-\partial^{\mu} h\right) \gamma_{\mu}\right] \\
& \overleftarrow{\mathcal{D}}=\hbar c\left[\left(i \gamma^{k} \overleftarrow{\partial_{k}}+m c / \hbar\right)\left(1+\frac{1}{2} h\right)-\frac{i}{2} \overleftarrow{\partial_{k}} \gamma_{\mu} h^{\mu k}-\frac{i}{4}\left(\partial_{\nu} h^{\mu \nu}-\partial^{\mu} h\right) \gamma_{\mu}\right] .
\end{aligned}
$$

One then finds that the Hamiltonian density $\Pi \dot{\Psi}-\mathcal{L}$ can be written as

$$
\mathcal{H}=\frac{1}{2}[\bar{\Psi} \overrightarrow{\mathcal{D}} \Psi+\bar{\Psi} \overleftarrow{\mathcal{D}} \Psi]
$$

while the field equation reads

$$
i \hbar c \gamma^{-1} \partial_{0} \Psi=\overrightarrow{\mathcal{D}} \Psi
$$

The relation $\overline{\overline{\mathcal{D}} \Psi}=\bar{\Psi} \overleftarrow{\mathcal{D}}$ and the formula $\left(\gamma^{-1}\right)^{\dagger}=\gamma^{0} \gamma^{-1} \gamma^{0}$ then give the conjugate equation

$$
-i \hbar c \partial_{0} \bar{\Psi} \gamma^{-1}=\bar{\Psi} \overline{\mathcal{D}}
$$

Using an integration by parts, the total Hamiltonian $H$ can be transformed into

$$
H=\int\left(d^{3} x\right) \bar{\Psi} \overrightarrow{\mathcal{D}} \Psi+\frac{i \hbar c}{2} \int\left(d^{3} x\right) \bar{\Psi}\left(\partial_{0} \gamma^{-1}\right) \Psi .
$$

The anticommutation relation (17) then gives the commutator

$$
[H, \Psi]=-\gamma \overrightarrow{\mathcal{D}} \Psi-\frac{i}{2} \hbar c\left(\gamma \partial_{0} \gamma^{-1}\right) \Psi
$$

or, given the field equation,

$$
[H, \Psi]=-i \hbar c \partial_{0} \Psi-\frac{i}{2} \hbar c\left(\gamma \partial_{0} \gamma^{-1}\right) \Psi
$$

Similarly, one has

$$
[H, \bar{\Psi}]=-i \hbar c \partial_{0} \bar{\Psi}-\frac{i}{2} \hbar c \bar{\Psi}\left(\partial_{0} \gamma^{-1}\right) \gamma
$$

then

$$
[H, \Pi]=-i \hbar c \partial_{0} \Pi+\frac{i}{2} \hbar c \Pi \gamma \partial_{0} \gamma^{-1} .
$$

The second terms in the right members of (25), (26) and (27) are unusual, since the fields $\Psi, \bar{\Psi}$ and $\Pi$ behave as if, considered as functions of some fundamental dynamical variables, they were also explicitly dependent on the time. From the expression of $\Pi$, it is in fact obvious that, among $\bar{\Psi}$ and $\Pi$ one of them, at least, explicitly depends on time. But this is not obvious for $\Psi$. 


\subsubsection{Asymmetrical Lagrangian.}

The preceding difficulty takes another form if, as it is more usual [18], the Lagrangian (4) is replaced by the asymmetrical one

$$
\begin{aligned}
\mathcal{L}^{\prime} & =\mathcal{L}+\frac{i \hbar}{2} \partial_{\mu} j^{\mu} \\
& =\hbar c \bar{\Psi}\left[\left(1+\frac{1}{2} h\right)\left(i \gamma^{\mu} \overrightarrow{\partial_{\mu}}-m c / \hbar\right) \Psi-\frac{i}{2} h^{\mu \nu} \gamma_{\mu} \overrightarrow{\partial_{\nu}} \Psi-\frac{i}{4} \partial_{\nu} h^{\mu \nu} \gamma_{\mu} \Psi+\frac{i}{4} \partial_{\mu} h \gamma^{\mu} \Psi\right]
\end{aligned}
$$

The field equations are left unchanged, and the conjugate field is again:

$$
\Pi^{\prime}=\frac{\partial \mathcal{L}^{\prime}}{\partial \dot{\Psi}}=i \hbar \bar{\Psi} \gamma^{-1}
$$

Since $\Pi^{\prime}=\Pi$, the anticommutation relation of $\Psi$ and $\bar{\Psi}$ is identical to (17), but the Hamiltonian is now

$$
H^{\prime}=\int\left(d^{3} x\right) \bar{\Psi} \overrightarrow{\mathcal{D}} \Psi=\int\left(d^{3} x\right) \bar{\Psi} \overleftarrow{\mathcal{D}} \Psi-i \hbar c \int\left(d^{3} x\right) \bar{\Psi}\left(\partial_{0} \gamma^{-1}\right) \Psi
$$

It follows that the usual Heisenberg equations are satisfied. In fact, one has

$$
\begin{aligned}
& {\left[H^{\prime}, \Psi\right]=-\gamma \overrightarrow{\mathcal{D}} \Psi=-i \hbar c \partial_{0} \Psi} \\
& {\left[H^{\prime}, \bar{\Psi}\right]=\bar{\Psi} \overline{\mathcal{D}} \gamma-i \hbar c \bar{\Psi}\left(\partial_{0} \gamma^{-1}\right) \gamma=-i \hbar c \partial_{0} \bar{\Psi}-i \hbar c \bar{\Psi}\left(\partial_{0} \gamma^{-1}\right) \gamma}
\end{aligned}
$$

then

$$
\left[H^{\prime}, \Pi^{\prime}\right]=-i \hbar c \partial_{0} \Pi^{\prime}
$$

The equations satisfied by $\Psi$ and $\Pi^{\prime}$ are those of variables having no explicit time dependence, which is the rule for canonical variables. On the contrary, expression (29) shows that $\bar{\Psi}$ depends explicitly on time since $\gamma^{-1}$ does, and equation (32) is in agreement with this dependence.

However, the Hamiltonian $H^{\prime}$ is not Hermitian. In fact, since one has $(\bar{\Psi} \overrightarrow{\mathcal{D}} \Psi)^{\dagger}=\bar{\Psi} \overline{\mathcal{D}} \Psi$, it follows that

$$
H^{\prime \dagger}=\int\left(d^{3} x\right) \bar{\Psi} \overline{\mathcal{D}} \Psi=H^{\prime}+i \hbar c \int\left(d^{3} x\right) \bar{\Psi}\left(\partial_{0} \gamma^{-1}\right) \Psi
$$

The asymmetry of the equations for $\Psi$ and $\bar{\Psi}$ is a consequence of this lack of Hermiticity.

\subsubsection{Trouble with the interaction picture.}

A common shortcoming of the two preceding methods of quantization is the absence of a coherent definition of the interaction picture. In fact, if this picture was defined, there would be a unitary operator $U$, such that the corresponding field variables $\psi$ and $\varpi$ would be given by

$$
\psi=U \Psi U^{-1}, \quad \varpi=U \Pi U^{-1} .
$$

Moreover, these variables would be free fields so that one would have the relation $\varpi=i \hbar \bar{\psi} \gamma_{0}$. Such a relation is not compatible with (35) since one has $\Pi=i \hbar \bar{\Psi} \gamma^{-1}, \gamma^{-1} \neq \gamma_{0}$, and since $U$ must be unitary.

In what follows, the quantization is defined in a way such that the preceding difficulties do not appear. In particular, the interaction picture will be defined, allowing the construction of the transition probabilities and that of the $\mathrm{S}$ matrix. 


\subsection{A coherent method of quantization}

The afore-mentioned problems will be solved by a change of variables eliminating the derivative coupling. The Lagrangian density (44) can be written under the form

$$
\mathcal{L}=\frac{i \hbar c}{2}\left(\bar{\Psi} \gamma^{-1}\left(\partial_{0} \Psi\right)-\left(\partial_{0} \bar{\Psi}\right) \gamma^{-1} \Psi\right)-\frac{1}{2} \bar{\Psi}(\overrightarrow{\mathcal{D}}+\overline{\mathcal{D}}) \Psi
$$

Let us introduce the new field $\Theta$ by the formula $\Psi=\Lambda \Theta$, where $\Lambda$ is a matrix to be determined, which depends on the coordinates. One has

$$
\bar{\Psi} \gamma^{-1}\left(\partial_{0} \Psi\right)=\bar{\Theta} \gamma^{0} \Lambda^{\dagger} \gamma^{0} \gamma^{-1} \Lambda\left(\partial_{0} \Theta\right)+\bar{\Theta} \gamma^{0} \Lambda^{\dagger} \gamma^{0} \gamma^{-1}\left(\partial_{0} \Lambda\right) \Theta
$$

The terms containing the time derivatives of $\Theta$ and $\bar{\Theta}$ in $(36)$ will be those of the free Dirac Lagrangian if one has $\gamma^{0} \Lambda^{\dagger} \gamma^{0} \gamma^{-1} \Lambda=\gamma^{0}$ or, assuming the inversibility of $\Lambda$, if $\gamma^{0} \gamma^{-1}=\Lambda^{\dagger-1} \Lambda^{-1}$. By writing the matrix $\Lambda$ under the form $\Lambda=M U$ where $M$ is Hermitian and positive [34] and $U$ unitary, the preceding equation becomes

$$
\gamma^{0} \gamma^{-1}=\left(M^{-1}\right)^{2}=I+\frac{1}{2} h-\frac{1}{2} h^{0 \mu} \gamma^{0} \gamma_{\mu}
$$

If $h_{\mu \nu}$ is sufficiently small, this matrix is inversible and defines a unique matrix $M$ positive-definite [34], while $U$ may be arbitrary. However, to ensure that the asymptotic states deduced from $\Theta$ or $\Psi$ have the same physical interpretation, it is necessary that $U$ goes to the identity when $t \rightarrow \pm \infty$. More precisely, we will impose the condition $U=I$, by which, when $h_{\mu \nu}=0$, the field $\Theta$ is a free field as $\Psi$.

The Lagrangian density takes the following form as a function of $\Theta$ :

$$
\mathcal{L}=\hbar c\left[\frac{i}{2}\left(\bar{\Theta} \gamma^{0}\left(\partial_{0} \Theta\right)-\left(\partial_{0} \bar{\Theta}\right) \gamma^{0} \Theta\right)+\frac{i}{2}\left(\bar{\Theta} \Gamma^{k}\left(\partial_{k} \Theta\right)-\left(\partial_{k} \bar{\Theta}\right) \Gamma^{k} \Theta\right)+\frac{1}{2} \bar{\Theta} \Gamma \Theta\right]
$$

where $\Gamma^{k}$ and $\Gamma$ are defined by

$$
\begin{aligned}
\Gamma^{k}= & \gamma^{0} \Lambda^{\dagger} \gamma^{0}\left[\left(1+\frac{1}{2} h\right) \gamma^{k}-\frac{1}{2} h^{\mu k} \gamma_{\mu}\right] \Lambda, \\
\Gamma= & i\left(\gamma^{0} \Lambda^{-1} \partial_{0} \Lambda+\Gamma^{k} \Lambda^{-1} \partial_{k} \Lambda\right) \\
& -i \gamma^{0}\left(\left(\partial_{0} \Lambda^{\dagger}\right) \Lambda^{\dagger-1} \gamma^{0}+\left(\partial_{k} \Lambda^{\dagger}\right) \Lambda^{\dagger-1}\left(\gamma^{0} \Gamma^{k} \gamma^{0}\right)\right) \gamma^{0}-2 \frac{m c}{\hbar}\left(1+\frac{1}{2} h\right) \gamma^{0} \Lambda^{\dagger} \gamma^{0} \Lambda
\end{aligned}
$$

Let us note the Hermiticity relations

$$
\left(\Gamma^{k}\right)^{\dagger}=\gamma^{0} \Gamma^{k} \gamma^{0}, \quad \Gamma^{\dagger}=\gamma^{0} \Gamma \gamma^{0}
$$

The field equations, equivalent to those of $\Psi$ and $\bar{\Psi}$, are now

$$
\begin{aligned}
i\left(\gamma^{0}\left(\partial_{0} \Theta\right)+\Gamma^{k}\left(\partial_{k} \Theta\right)\right)+\frac{1}{2}\left(\Gamma+i\left(\partial_{k} \Gamma^{k}\right)\right) \Theta & =0, \\
-i\left(\left(\partial_{0} \bar{\Theta}\right) \gamma^{0}+\left(\partial_{k} \bar{\Theta}\right) \Gamma^{k}\right)+\frac{1}{2} \bar{\Theta}\left(\Gamma-i\left(\partial_{k} \Gamma^{k}\right)\right) & =0,
\end{aligned}
$$

while the current reads

$$
j^{\mu}=c \bar{\Theta} \gamma^{0} \Lambda^{\dagger} \gamma^{0}\left[\left(1+\frac{1}{2} h\right) \gamma^{\mu}-\frac{1}{2} h^{\mu \nu} \gamma_{\nu}\right] \Lambda \Theta
$$

or, more explicitlyÐ

$$
j^{0}=c \bar{\Theta} \gamma^{0} \Theta, \quad j^{k}=c \bar{\Theta} \Gamma^{k} \Theta .
$$

\footnotetext{
${ }^{5}$ The expression of $j^{0}$ is identical to that of the free-field case. In Appendix B this property is taken as a condition allowing the introduction of the field $\Theta$ in the framework of the linearized theory of General Relativity.
} 
The conjugate momentum $\Pi_{\Theta}$ of $\Theta$ has the same form as in the free-field case

$$
\Pi_{\Theta}=2 \frac{\partial \mathcal{L}}{\partial \dot{\Theta}}=i \hbar \bar{\Theta} \gamma^{0}
$$

It follows that the anticommutation relation of $\Theta$ and $\bar{\Theta}$ is the usual one

$$
\left\{\Theta_{\alpha}(x), \bar{\Theta}_{\beta}(y)\right\}_{x^{0}=y^{0}}=\gamma_{\alpha \beta}^{0} \delta(\vec{x}-\vec{y}) .
$$

It is equivalent to the anticommutation relation (17) of $\Psi$ and $\bar{\Psi}$. The Hamiltonian density $\mathcal{H}_{\Theta}$ now reads

$$
\mathcal{H}_{\Theta}=-\frac{i \hbar c}{2}\left(\bar{\Theta} \Gamma^{k}\left(\partial_{k} \Theta\right)-\left(\partial_{k} \bar{\Theta}\right) \Gamma^{k} \Theta\right)-\frac{\hbar c}{2} \bar{\Theta} \Gamma \Theta
$$

which gives for the total Hamiltonian

$$
H_{\Theta}=-\frac{\hbar c}{2} \int\left(d^{3} x\right)\left[i\left(\bar{\Theta} \Gamma^{k}\left(\partial_{k} \Theta\right)-\left(\partial_{k} \bar{\Theta}\right) \Gamma^{k} \Theta\right)+\bar{\Theta} \Gamma \Theta\right]
$$

or, equivalently,

$$
\begin{aligned}
H_{\Theta} & =-\hbar c \int\left(d^{3} x\right) \bar{\Theta}\left[i \Gamma^{k}\left(\partial_{k} \Theta\right)+\frac{1}{2}\left(\Gamma+i\left(\partial_{k} \Gamma^{k}\right)\right) \Theta\right] \\
& =\hbar c \int\left(d^{3} x\right)\left[i\left(\partial_{k} \bar{\Theta}\right) \Gamma^{k}-\frac{1}{2} \bar{\Theta}\left(\Gamma-i\left(\partial_{k} \Gamma^{k}\right)\right)\right] \Theta .
\end{aligned}
$$

This operator is Hermitian, and from the preceding expressions and, from the field equations (43) and (44), one checks the Heisenberg equations

$$
\left[H_{\Theta}, \Theta\right]=-i \hbar c \partial_{0} \Theta, \quad\left[H_{\Theta}, \bar{\Theta}\right]=-i \hbar c \partial_{0} \bar{\Theta}, \quad\left[H_{\Theta}, \Pi_{\Theta}\right]=-i \hbar c \partial_{0} \Pi_{\Theta}
$$

The difficulties discussed in Section 3.1 have disappeared. In particular, the basic variables $\Theta, \bar{\Theta}$ and $\Pi_{\Theta}$ look like variables having no explicit dependence on time. On the contrary, the variables $\Psi, \bar{\Psi}$ and $\Pi$, initially considered, depend explicitly on time, since one has

$$
\Psi=\Lambda \Theta, \quad \bar{\Psi}=\bar{\Theta} \gamma^{0} \Lambda^{\dagger} \gamma^{0}, \quad \Pi=\Pi_{\Theta} \Lambda^{-1},
$$

and since the matrix $\Lambda$ generally depends on time.

Let us remark, however, that the Hamiltonian $H_{\Theta}$ differs from the Hamiltonian $H$ introduced at the beginning in terms of $\Psi$. It is convenient to consider $H$ from (23) as the integral of the density

$$
\mathcal{H}_{\Psi}=\bar{\Psi}\left[\overrightarrow{\mathcal{D}}+\frac{i \hbar c}{2}\left(\partial_{0} \gamma^{-1}\right)\right] \Psi
$$

and $H_{\Theta}$ from (51) as the integral of the density

$$
\mathcal{H}_{\Theta}=\hbar c \bar{\Theta}\left[-i \Gamma^{k} \overrightarrow{\partial_{k}}-\frac{1}{2}\left(\Gamma+i\left(\partial_{k} \Gamma^{k}\right)\right)\right] \Theta
$$

A rather tedious transformation of the former expression, using the expressions of $\gamma^{-1}, \Gamma^{k}, \overrightarrow{\mathcal{D}}$, and the definition of $\Theta$, leads to the formula

$$
\mathcal{H}_{\Psi}=\mathcal{H}_{\Theta}+\frac{i \hbar c}{2} \bar{\Theta} \gamma^{0}\left[\Lambda^{-1}\left(\partial_{0} \Lambda\right)-\left(\partial_{0} \Lambda^{\dagger}\right) \Lambda^{\dagger-1}\right] \Theta .
$$

Conversely, one has

$$
\mathcal{H}_{\Theta}=\mathcal{H}_{\Psi}+\frac{i \hbar c}{2} \bar{\Psi} \gamma^{0}\left[\Lambda^{\dagger-1}\left(\partial_{0} \Lambda^{-1}\right)-\left(\partial_{0} \Lambda^{\dagger-1}\right) \Lambda^{-1}\right] \Psi
$$


With the choice made above of the matrix $U$, one has $\Lambda=M=M^{\dagger}$, with $M$ defined by (38). The two preceding formulas can then be written

$$
\begin{aligned}
& \mathcal{H}_{\Psi}=\mathcal{H}_{\Theta}+\frac{i \hbar c}{2} \bar{\Theta} \gamma^{0}\left[M^{-1}, \partial_{0} M\right] \Theta, \\
& \mathcal{H}_{\Theta}=\mathcal{H}_{\Psi}+\frac{i \hbar c}{2} \bar{\Psi} \gamma^{0}\left[M^{-1}, \partial_{0} M^{-1}\right] \Psi .
\end{aligned}
$$

It is noticeable that the equality $\mathcal{H}_{\Psi}=\mathcal{H}_{\Theta}$ is valid at first order with respect to the $h_{\mu \nu}$ 's. In fact, since $\partial_{0} M$ and $\partial_{0} M^{-1}$ are first-order quantities, this approximation is obtained by taking, for the first term in the commutators in (59) and (60), the zeroth-order approximation of $M^{-1}$, that is the matrix unity.

\section{Interaction picture and the $S$-matrix}

With the Lagrangian (39) the interaction picture is easily defined, since the corresponding conjugate momentum $\Pi_{\Theta}$ has the same form as that of the free theory. This allows us to define the evolution operator in that picture, then the $S$-matrix.

\subsection{Evolution operator and transition amplitudes}

The field in the interaction picture will be denoted by $\theta$. Let us recall that this operator is obtained from the Heisenberg operator $\Theta$ by a unitary transformation such that the field equation becomes the free one [32]. Accordingly, the evolution equation of the state vector reads

$$
i \hbar \frac{d}{d t}|\Phi(t)\rangle=H_{I}(t)|\Phi(t)\rangle
$$

where, in the absence of derivative coupling, which is the case for the Lagrangian (39), the Hamiltonian $H_{I}(t)$ is equal to the interaction Hamiltonian expressed in terms of $\theta$. From the expression (50) of the total Hamiltonian one gets

$$
H_{I}(t)=\int\left(d^{3} x\right) \mathcal{H}_{\text {int }}(x)
$$

with

$$
\mathcal{H}_{\text {int }}=-\frac{i \hbar c}{2} \bar{\theta}\left(\Gamma^{k}-\gamma^{k}\right)\left(\partial_{k} \theta\right)+\frac{i \hbar c}{2}\left(\partial_{k} \bar{\theta}\right)\left(\Gamma^{k}-\gamma^{k}\right) \theta-\frac{\hbar c}{2} \bar{\theta}\left(\Gamma+2 \frac{m c}{\hbar}\right) \theta
$$

The evolution operator in the interaction picture is, from (61), the solution of the following equation together with the initial condition [21] :

$$
i \hbar \frac{d}{d t} U\left(t, t_{0}\right)=H_{I}(t) U\left(t, t_{0}\right), \quad U\left(t_{0}, t_{0}\right)=I
$$

The perturbation theory is then obtained from the integral equation, equivalent to (64),

$$
U\left(t, t_{0}\right)=I-\frac{i}{\hbar} \int_{t_{0}}^{t} H_{I}(\tau) U\left(\tau, t_{0}\right) d \tau
$$

In what follows, we are interested in the transition amplitudes to first order with respect to the $h_{\mu \nu}$ 's. These amplitudes will be obtained from the first-order approximation with respect to the Hamiltonian $H_{I}$ of the $U$-operator, namely

$$
U^{(1)}\left(t, t_{0}\right)=-\frac{i}{\hbar} \int_{t_{0}}^{t} H_{I}(\tau) d \tau .
$$


By introducing the Hamiltonian density (63) in normal form transition, we will consider the amplitudes

$$
\left\langle\Phi_{f}\left|U^{(1)}\left(t, t_{0}\right)\right| \Phi_{i}\right\rangle=-\frac{i}{\hbar c} \int_{t_{0}}^{t}\left(d^{4} x\right)\left\langle\Phi_{f}\left|: \mathcal{H}_{\mathrm{int}}(x):\right| \Phi_{i}\right\rangle,
$$

where the pair of colons : : denotes as usual the normal product.

The first-order expressions needed for the evaluation of (67) are now derived from (38) which gives

$$
\Lambda=M=I-\frac{h}{4}+\frac{1}{4} h^{0 \mu} \gamma^{0} \gamma_{\mu}
$$

then, from (40) and (41),

$$
\begin{aligned}
\Gamma^{k}-\gamma^{k} & =\frac{1}{2}\left[h^{00} \gamma^{k}-h^{0 k} \gamma^{0}-h^{\mu k} \gamma_{\mu}\right] \\
\Gamma+2 \frac{m c}{\hbar} & =-\frac{m c}{\hbar} h^{00}+\frac{i}{4}\left(\partial_{k} h^{0 \mu}\right)\left(\gamma^{k} \gamma^{0} \gamma_{\mu}-\gamma_{\mu} \gamma^{0} \gamma^{k}\right) .
\end{aligned}
$$

The corresponding expression for the Hamiltonian density $\mathcal{H}_{\text {int }}$ is

$$
\begin{aligned}
\mathcal{H}_{\text {int }}= & -\frac{i \hbar c}{4} \bar{\theta}\left[h^{00} \gamma^{k}-h^{0 k} \gamma^{0}-h^{\mu k} \gamma_{\mu}\right]\left(\partial_{k} \theta\right) \\
& +\frac{i \hbar c}{4}\left(\partial_{k} \bar{\theta}\right)\left[h^{00} \gamma^{k}-h^{0 k} \gamma^{0}-h^{\mu k} \gamma_{\mu}\right] \theta \\
& +\frac{\hbar c}{2} \bar{\theta}\left[\frac{m c}{\hbar} h^{00}-\frac{i}{4}\left(\partial_{k} h^{0 \mu}\right)\left(\gamma^{k} \gamma^{0} \gamma_{\mu}-\gamma_{\mu} \gamma^{0} \gamma^{k}\right)\right] \theta
\end{aligned}
$$

In Section 5, the expression of the Hamiltonian $H_{I}(t)$ which will be used is the expression obtained from the space integral of (71) by performing the integration by parts of the term containing $\partial_{k} \bar{\theta}$, which gives

$$
H_{I}(t)=\int\left(d^{3} x\right) \overline{\theta(x)} \gamma^{0} \mathcal{V}_{G}(x) \theta(x)
$$

where the operator $\mathcal{V}_{G}(x)$, acting on $\theta(x)$, is given by

$$
\begin{aligned}
\mathcal{V}_{G}(x)= & \frac{\hbar c}{2} \gamma^{0}\left[\frac{m c}{\hbar} h^{00}+\frac{i}{4} \partial_{k} h_{0 j} \gamma^{0}\left(\gamma^{k} \gamma^{j}-\gamma^{j} \gamma^{k}\right)\right. \\
& \left.+\frac{i}{2}\left(2 \partial_{k} h^{0 k} \gamma^{0}+\partial_{k} h^{j k} \gamma_{j}-\partial_{k} h^{00} \gamma^{k}\right)\right] \\
+ & \frac{i \hbar c}{2} \gamma^{0}\left[2 h^{0 k} \gamma^{0}+h^{j k} \gamma_{j}-h^{00} \gamma^{k}\right] \partial_{k} .
\end{aligned}
$$

This form of the Hamiltonian is closely related to the equation of motion (43) of the Heisenberg field $\Theta$. In fact, with the help of (69) and (70), one checks that this equation can be written:

$$
\left(i \hbar c \gamma^{\mu} \partial_{\mu}-m c^{2}\right) \Theta=\gamma^{0} \mathcal{V}_{G} \Theta
$$

In what follows, the initial and final states, which appear in (67), are some one-particle or antiparticle states. They are defined from positive or negative energy solutions $\chi_{i}$ and $\chi_{f}$ of the free Dirac equation

$$
\left(i \gamma^{\mu} \overrightarrow{\partial_{\mu}}-m c / \hbar\right) \chi_{k}=0, \quad \overline{\chi_{k}}\left(i \gamma^{\mu} \overleftarrow{\partial_{\mu}}+m c / \hbar\right)=0, \quad k=i, f
$$

\footnotetext{
${ }^{6}$ See [21]. To first order this prescription suppresses an infinite contribution, due to the energy of the vacuum, in the transition amplitudes for the antiparticles only. It can be seen, therefore, as an expression of the symmetry between particles and antiparticles.
} 
Denoting by $\left|\chi_{k}\right\rangle$ the corresponding states, one has then to calculate the matrix element

$$
\left\langle\chi_{f}\left|: \overline{\theta(x)} \gamma^{0} \mathcal{V}_{G}(x) \theta(x):\right| \chi_{i}\right\rangle
$$

As a free field, the operator $\theta$ can be written

$$
\theta(x)=\sum_{r=1}^{2} \int\left(d^{3} p\right)\left[b_{r}(\vec{p}) \chi_{\vec{p}, r}^{(+)}(x)+d_{r}^{\dagger}(\vec{p}) \chi_{\vec{p}, r}^{(-)}(x)\right],
$$

where $b_{r}(\vec{p})$ and $d_{r}(\vec{p})$ are the annihilation operators for the particles or antiparticles, respectively, and $\chi_{\vec{p}, r}^{( \pm)}$the positive or negative energy solutions of the free Dirac equation given by [17, 21]

$$
\begin{aligned}
& \chi_{\vec{p}, r}^{(+)}(x)=\frac{1}{(2 \pi \hbar)^{3 / 2}} \sqrt{\frac{m c^{2}}{E(\vec{p})}} u^{(r)}(\vec{p}) e^{i(\vec{p} \cdot \vec{x}-E(\vec{p}) t) / \hbar}, \\
& \chi_{\vec{p}, r}^{(-)}(x)=\frac{1}{(2 \pi \hbar)^{3 / 2}} \sqrt{\frac{m c^{2}}{E(\vec{p})}} v^{(r)}(\vec{p}) e^{-i(\vec{p} \cdot \vec{x}-E(\vec{p}) t) / \hbar},
\end{aligned}
$$

with $E(\vec{p})=c \sqrt{p^{2}+m^{2} c^{2}}, p=\|\vec{p}\|$. In terms of these, any solution $\chi$ with positive or negative energy can be written

$$
\chi(x)=\sum_{r} \int\left(d^{3} p\right) \chi_{\vec{p}, r}^{( \pm)}(x)\left(\chi_{\vec{p}, r}^{( \pm)}, \chi\right),
$$

the scalar product of two solutions being defined by

$$
\left(\chi_{1}, \chi_{2}\right)=\int\left(d^{3} x\right) \overline{\chi_{1}}(x) \gamma^{0} \chi_{2}(x)
$$

From (80) we have the following expression of the one-particle states

$$
|\chi\rangle=\sum_{r} \int\left(d^{3} p\right)\left(\chi_{\vec{p}, r}^{(+)}, \chi\right) b_{r}^{\dagger}(\vec{p})\left|\phi_{0}\right\rangle,
$$

where $\left|\phi_{0}\right\rangle$ is the vacuum state, the correspondence $\chi \rightarrow|\chi\rangle$ preserving the scalar product. Denoting by $\theta^{(+)}$the positive frequency part of $\theta$, this last formula implies

$$
\begin{aligned}
\theta^{(+)}(x)|\chi\rangle & =\sum_{r} \int\left(d^{3} p\right) \chi_{\vec{p}, r}^{(+)}(x) b_{r}(\vec{p})|\chi\rangle \\
& =\sum_{r} \int\left(d^{3} p\right) \chi_{\vec{p}, r}^{(+)}(x)\left(\chi_{\vec{p}, r}^{(+)}, \chi\right)\left|\phi_{0}\right\rangle \\
& =\chi(x)\left|\phi_{0}\right\rangle
\end{aligned}
$$

The matrix element (76) reduces to

$$
\left\langle\chi_{f}\left|\overline{\theta^{(+)}(x)} \gamma^{0} \mathcal{V}_{G}(x) \theta^{(+)}(x)\right| \chi_{i}\right\rangle=\overline{\chi_{f}(x)} \gamma^{0} \mathcal{V}_{G}(x) \chi_{i}(x),
$$

so that, from (72), the amplitude (67) reads

$$
\left\langle\chi_{f}\left|U^{(1)}\left(t, t_{0}\right)\right| \chi_{i}\right\rangle=-\frac{i}{\hbar c} \int_{t_{0}}^{t}\left(d^{4} x\right) \overline{\chi_{f}(x)} \gamma^{0} \mathcal{V}_{G}(x) \chi_{i}(x)
$$

This formula will be analyzed in more detail in Section 5. 


\subsection{S-matrix}

The $S$-matrix can be defined when the external field vanishes at the limit of infinite time7. It is obtained from the $U$ operator by taking the limits $t_{0} \rightarrow-\infty$ and $t \rightarrow+\infty$, and the corresponding amplitudes can then be given from (85). However, it is convenient here to return to the formula (67) in which the Hamiltonian density is given by (71). In fact, we want to show that this expression can be transformed into a covariant one, the expression already given by Gupta [16].

The initial and final states being the same as in the calculation leading to (85), formula (67) with the expression $(71)$ of $\mathcal{H}_{\text {int }}$ gives, for the first-order $S$-matrix,

$$
\begin{aligned}
\left\langle\chi_{f}\left|S^{(1)}\right| \chi_{i}\right\rangle= & -i \int\left(d^{4} x\right)\left\{\frac{1}{2} \overline{\chi_{f}}\left[\frac{m c}{\hbar} h^{00}-\frac{i}{4}\left(\partial_{k} h^{0 \mu}\right)\left(\gamma^{k} \gamma^{0} \gamma_{\mu}-\gamma_{\mu} \gamma^{0} \gamma^{k}\right)\right] \chi_{i}\right. \\
& +\frac{i}{4} \overline{\chi_{f}}\left[h^{\mu k} \gamma_{\mu}+h^{0 k} \gamma^{0}-h^{00} \gamma^{k}\right]\left(\partial_{k} \chi_{i}\right) \\
& \left.-\frac{i}{4}\left(\partial_{k} \overline{\chi_{f}}\right)\left[h^{\mu k} \gamma_{\mu}+h^{0 k} \gamma^{0}-h^{00} \gamma^{k}\right] \chi_{i}\right\}
\end{aligned}
$$

This expression can be simplified by integrating by parts the term containing the derivative $\partial_{k} h^{0 \mu}$. This leads to the two terms

$$
\frac{i}{8} h^{0 \mu}\left[\left(\partial_{k} \overline{\chi_{f}}\right)\left(\gamma^{k} \gamma^{0} \gamma_{\mu}-\gamma_{\mu} \gamma^{0} \gamma^{k}\right) \chi_{i}+\overline{\chi_{f}}\left(\gamma^{k} \gamma^{0} \gamma_{\mu}-\gamma_{\mu} \gamma^{0} \gamma^{k}\right)\left(\partial_{k} \chi_{i}\right)\right]
$$

In each of these, by introducing either of the formulas

$$
\begin{aligned}
\gamma^{k} \gamma^{0} \gamma_{\mu}-\gamma_{\mu} \gamma^{0} \gamma^{k} & =2\left[\delta_{\mu}^{0} \gamma^{k}-\delta_{\mu}^{k} \gamma^{0}-\gamma_{\mu} \gamma^{0} \gamma^{k}\right] \\
& =2\left[\delta_{\mu}^{k} \gamma^{0}-\delta_{\mu}^{0} \gamma^{k}+\gamma^{k} \gamma^{0} \gamma_{\mu}\right]
\end{aligned}
$$

one can insert the derivatives in the combinations $\gamma^{k} \partial_{k} \chi_{i}$ or $\partial_{k} \overline{\chi_{f}} \gamma^{k}$, yielding

$$
\frac{i}{4} h^{0 k} \overline{\chi_{f}} \gamma^{0}\left(\overleftarrow{\partial_{k}}-\overrightarrow{\partial_{k}}\right) \chi_{i}+\frac{i}{4} h^{0 \mu}\left[\overline{\chi_{f}}\left(\delta_{\mu}^{0}-\gamma_{\mu} \gamma^{0}\right)\left(\gamma^{k} \partial_{k} \chi_{i}\right)-\left(\partial_{k} \overline{\chi_{f}} \gamma^{k}\right)\left(\delta_{\mu}^{0}-\gamma^{0} \gamma_{\mu}\right) \chi_{i}\right]
$$

Adding this contribution to the remaining terms in (86), one gets

$$
\begin{aligned}
\left\langle\chi_{f}\left|S^{(1)}\right| \chi_{i}\right\rangle=-i \int & \left(d^{4} x\right)\left\{\frac{m c}{2 \hbar} h^{00} \overline{\chi_{f}} \chi_{i}+\frac{i}{4} h^{\mu k} \overline{\chi_{f}} \gamma_{\mu}\left(\overrightarrow{\partial_{k}}-\overleftarrow{\partial_{k}}\right) \chi_{i}\right. \\
& \left.-\frac{i}{4} h^{\mu 0}\left[\overline{\chi_{f}} \gamma_{\mu} \gamma^{0}\left(\gamma^{k} \partial_{k} \chi_{i}\right)-\left(\partial_{k} \overline{\chi_{f}} \gamma^{k}\right) \gamma^{0} \gamma_{\mu} \chi_{i}\right]\right\} .
\end{aligned}
$$

Finally, using the Dirac equation in the last bracket, this expression reduces to

$$
\left\langle\chi_{f}\left|S^{(1)}\right| \chi_{i}\right\rangle=-i \int\left(d^{4} x\right) \frac{i}{4} h^{\mu \nu} \overline{\chi_{f}}\left(\gamma_{\mu} \overrightarrow{\partial_{\nu}}-\overleftarrow{\partial_{\nu}} \gamma_{\mu}\right) \chi_{i}
$$

This formula agrees with the rule given by Gupta [16] : the expression appearing under the integral sign is, up to the sign, obtained from the interaction Lagrangian $\mathcal{L}-\mathcal{L}_{0}$ in (41) by replacing $\Psi$ by $\chi_{i}$ and $\bar{\Psi}$ by $\overline{\chi_{f}}$. This result is identical with the one valid in the case of a nonderivative coupling, where the interaction picture exists directly. More generally, the validity of the rule asserted by Gupta, implying the use of the interaction Lagrangian defined by (四) and the covariant form of the propagators, can be proved at higher orders in the quantized theory defined in Section 3.

\footnotetext{
${ }^{7}$ Here, this condition is realized, for example, in the case of a gravitational wave.

${ }^{8}$ The contribution of the term $(h / 2) \mathcal{L}_{0}$ of (4) vanishes in the first order considered here, since $\chi_{i}$ and $\chi_{f}$ are solutions of the free Dirac equation. But this property is limited to the first order.
} 


\section{Calculation of the relativistic phase shifts in the weak-field ap- proximation}

In this final section we use the tools and the material derived in the previous sections to make an explicit calculation of the various contributions to a gravitationally induced phase shift in matterwave interferometry. We restrict ourselves to one-particle or one-antiparticle states. The application of the formalism to many-particle states and coherent beams of massive particles of different spins will be developed in another publication. We assume that the incoming particles or antiparticles are described by the state vector $\left|\chi\left(t_{0}\right)\right\rangle=\left|\chi_{i}\right\rangle$ at some time $t_{0}$ before interaction $\left(t_{0}\right.$ can be conveniently taken to be $-\infty)$. At a later time $t$, this state evolves into $|\chi(t)\rangle$ which interferes with a reference beam described by $\left|\chi_{\text {ref }}\right\rangle$ with which it is recombined in the final beam splitter. In practice, $\left|\chi_{\text {ref }}\right\rangle$ is produced by the other arm of the interferometer and in many cases, one will have $\left|\chi_{\text {ref }}\right\rangle \equiv\left|\chi_{i}\right\rangle$.

We are thus interested in the spinorial wave function for one-(anti)particle states:

$$
\chi(x)=\left\langle\phi_{0}|\theta(x)| \chi(t)\right\rangle
$$

where $\theta(x),|\chi(t)\rangle,\left|\phi_{0}\right\rangle$ are respectively the free-field operator, the one-(anti)particle and the vacuum state vectors in the interaction representation. It is easily shown that, when pair creations are neglected, this expression is equivalent to the Heisenberg field amplitude $\langle 0$, in $|\Theta(x)| \Phi\rangle$.

The interference signal itself is given by the projection:

$$
\begin{aligned}
\int d^{3} x \chi_{\mathrm{ref}}^{\dagger}(x) \chi(x) & =\int d^{3} x\left\langle\chi_{\mathrm{ref}}\left|\theta^{\dagger}(x)\right| \phi_{0}\right\rangle\left\langle\phi_{0}|\theta(x)| \chi(t)\right\rangle \\
& =\left\langle\chi_{\mathrm{ref}}\left|U\left(t, t_{0}\right)\right| \chi\left(t_{0}\right)\right\rangle
\end{aligned}
$$

where the space integral is over some detection volume. More generally one should consider a detection hypersurface $\sigma(x)$ and the projection:

$$
\int_{\sigma} d \sigma^{\mu} \bar{\chi}_{\mathrm{ref}}(x) \gamma_{\mu} \chi(x)=\int_{\sigma} d \sigma^{\mu}\left\langle\chi_{\mathrm{ref}}|\bar{\theta}(x)| \phi_{0}\right\rangle \gamma_{\mu}\left\langle\phi_{0}|\theta(x)| \chi(t)\right\rangle .
$$

In this paper, we shall limit ourselves to the calculation of the amplitude:

$$
\left\langle\chi_{\mathrm{ref}} \mid \chi(t)\right\rangle=\left\langle\chi_{\mathrm{ref}}\left|U\left(t, t_{0}\right)\right| \chi\left(t_{0}\right)\right\rangle \text {. }
$$

and take the phase of this complex amplitude as the phase contribution of the perturbing interaction. The resulting spinor (91) will also be derived using two different methods: first, in configuration space and second, in the momentum representation.

\subsection{Calculation in configuration space}

The evolution equation of the state vector in the interaction picture is

$$
i \hbar \frac{d}{d t}|\chi(t)\rangle=H_{I}(t)|\chi(t)\rangle
$$

where the Hamiltonian $H_{I}(t)$ is

$$
H_{I}(t)=\int d^{3} x \theta^{\dagger}(x) \mathcal{V}_{G}(x) \theta(x),
$$

and where the operator $\mathcal{V}_{G}(x)$, acting on the field operator $\theta(x)$, is given to first order by (73)

$$
\begin{aligned}
\mathcal{V}_{G}= & \frac{1}{2} m c^{2} \gamma^{0} h^{00}+\frac{i \hbar c}{8} \partial_{k} h_{0 j}\left(\gamma^{k} \gamma^{j}-\gamma^{j} \gamma^{k}\right) \\
& +\frac{i \hbar c}{4} \gamma^{0}\left(2 \partial_{k} h^{0 k} \gamma^{0}+\partial_{k} h^{j k} \gamma_{j}-\partial_{k} h^{00} \gamma^{k}\right) \\
& +\frac{i \hbar c}{2} \gamma^{0}\left[2 h^{0 k} \gamma^{0}+h^{j k} \gamma_{j}-h^{00} \gamma^{k}\right] \partial_{k}
\end{aligned}
$$


that we shall write:

$$
\mathcal{V}_{G}(x)=A(x)+\frac{i \hbar}{2} \partial_{j} B^{j}(x)+i \hbar B^{j}(x) \partial_{j}=A(x)+\frac{1}{2}\left\{i \hbar \partial_{j}, B^{j}(x)\right\}_{+}
$$

with:

$$
\begin{aligned}
A(x) & =\frac{1}{2} m c^{2} \gamma^{0} h^{00}+\frac{\hbar c}{4} \sigma^{k j} \partial_{k} h_{0 j} \\
B^{j}(x) & =\frac{c}{2} \gamma^{0}\left[2 h^{0 j} \gamma^{0}+h^{k j} \gamma_{k}-h^{00} \gamma^{j}\right] .
\end{aligned}
$$

From equation (91) we check that the evolution of the one-(anti)particle spinor is governed by the equation:

$$
i \hbar \partial_{t} \chi=-i \hbar c \gamma^{0} \gamma^{j} \partial_{j} \chi+m c^{2} \gamma^{0} \chi+\mathcal{V}_{G} \chi
$$

to which we may add terms corresponding to diagonal magnetic dipole and off-diagonal electric dipole interactions [10, 12]. This equation has been used in references [10] and [12] to discuss all the terms that lead to a phase shift in an interferometer.

To obtain the corresponding amplitude, we can start directly from the integral form of (95):

$$
|\chi(t)\rangle=\left|\chi\left(t_{0}\right)\right\rangle-\frac{i}{\hbar c} \int_{t_{0}}^{t} d^{4} x^{\prime} \theta^{\dagger}\left(x^{\prime}\right) \mathcal{V}_{G}\left(x^{\prime}\right) \theta\left(x^{\prime}\right)\left|\chi\left(t^{\prime}\right)\right\rangle
$$

So that, to first order:

$$
\begin{aligned}
\left\langle\chi_{\mathrm{ref}} \mid \chi^{(1)}(t)\right\rangle= & \left\langle\chi_{\mathrm{ref}}\left|U^{(1)}\left(t, t_{0}\right)\right| \chi_{i}\right\rangle \\
= & -\frac{i}{\hbar c} \int_{t_{0}}^{t} d^{4} x^{\prime} \chi_{\mathrm{ref}}^{\dagger}\left(x^{\prime}\right) \mathcal{V}_{G}\left(x^{\prime}\right) \chi_{i}\left(x^{\prime}\right) \\
= & -\frac{i}{\hbar c} \int_{t_{0}}^{t} d^{4} x\left\{\chi_{\mathrm{ref}}^{\dagger}\left[A(x)+\frac{i \hbar}{2} \partial_{j} B^{j}(x)+i \hbar B^{j}(x) \partial_{j}\right] \chi_{i}\right\} \\
= & \int_{t_{0}}^{t} d^{4} x\left\{\chi _ { \mathrm { ref } } ^ { \dagger } \left[\frac{h^{00}}{2 c} \gamma^{0} \partial_{t}+\vec{h} \cdot \vec{\nabla}-\frac{1}{2} \vec{\alpha} \cdot \vec{h} \cdot \vec{\nabla}\right.\right. \\
& \left.\left.+\frac{i}{4} \vec{\Sigma} \cdot \vec{\nabla} \times \vec{h}-\frac{1}{4}\left(\vec{\nabla} h^{00}+\vec{\nabla} \cdot \vec{h}\right) \cdot \vec{\alpha}+\frac{1}{2} \vec{\nabla} \cdot \vec{h}\right] \chi_{i}\right\}
\end{aligned}
$$

which follows also from Gupta's form. We have used the definitions:

$$
\vec{\alpha}=\gamma^{0} \vec{\gamma}=\left(\begin{array}{cc}
0 & \vec{\sigma} \\
\vec{\sigma} & 0
\end{array}\right), \vec{\Sigma}=\left(\begin{array}{cc}
\vec{\sigma} & 0 \\
0 & \vec{\sigma}
\end{array}\right), \vec{h}=\left\{h^{0 k}\right\}, \vec{h}=\left\{h^{i j}\right\}
$$

The calculation of the spinor itself, by a stationary phase method in configuration space, is outlined in Appendix C.

In equation (102), the first three terms lead to the familiar phase shifts of the Linet-Tourrenc formula [23], the fourth term is the spin-rotation interaction and the last two terms ensure hermiticity. But, because of the Dirac matrices, the interpretation of the various terms in configuration space is not so transparent and, in previous works non-relativistic limits have been taken either directly as in our own work [12] or through a Foldy-Wouthuysen transformation as in [13] in the case of inertial fields, to put equations (100) and (102) in a form where the significance of the terms is more obvious.

In this paper, we may as well take advantage of the flat Minkowski space-time and, therefore, we will use rather the momentum representation in the following. As we shall see, the interpretation of the terms is then much easier, even in their relativistic form. 


\subsection{Calculation in the momentum representation}

The free-field operator $\theta$ is written as before as:

$$
\theta(x)=\sum_{r=1}^{2} \int d^{3} p\left[b_{r}(\vec{p}) \chi_{\vec{p}, r}^{(+)}(x)+d_{r}^{\dagger}(\vec{p}) \chi_{\vec{p}, r}^{(-)}(x)\right],
$$

where $b_{r}(\vec{p})$ and $d_{r}(\vec{p})$ are the annihilation operators for the particles or antiparticles, respectively, and $\chi_{\vec{p}, r}^{( \pm)}$are the positive or negative energy solutions of the free Dirac equation given by (78) and (79).

Let us introduce the Fourier transforms $\widetilde{A}(\vec{k}, t), \widetilde{B}^{j}(\vec{k}, t), \widetilde{h}_{\mu \nu}(\vec{k})$ :

$$
\begin{aligned}
A(x) & =\frac{1}{(2 \pi)^{3 / 2}} \int d^{3} k \widetilde{A}(\vec{k}, t) e^{i \vec{k} \cdot \vec{x}} \\
B^{j}(x) & =\frac{1}{(2 \pi)^{3 / 2}} \int d^{3} k \widetilde{B}^{j}(\vec{k}, t) e^{i \vec{k} \cdot \vec{x}} \\
h_{\mu \nu}(x) & =\frac{1}{(2 \pi)^{3 / 2}} \int d^{3} k \widetilde{h}_{\mu \nu}(\vec{k}, t) e^{i \vec{k} \cdot \vec{x}} \\
\mathcal{V}_{G}(x) e^{i \vec{p} \cdot \vec{x} / \hbar} & =\frac{1}{(2 \pi)^{3 / 2}} \int d^{3} k \widetilde{\mathcal{V}}_{G}(\vec{k}, \vec{p}, t) e^{i \vec{k} \cdot \vec{x}} e^{i \vec{p} \cdot \vec{x} / \hbar} \\
\widetilde{\mathcal{V}_{G}}(\vec{k}, \vec{p}, t) & =\widetilde{A}(\vec{k}, t)-\widetilde{\vec{B}}(\vec{k}, t) \cdot\left(\vec{p}+\frac{1}{2} \hbar \vec{k}\right) .
\end{aligned}
$$

Let us expand $\chi(x), \overline{\chi_{\text {ref }}}(x)$ and $\chi_{i}(x)$ in plane waves using the expansions of $\overline{\theta^{( \pm)}}(x)$ and $\theta^{( \pm)}(x)$. For particles, the output spinor is:

$$
\begin{aligned}
\chi(x) & =\left\langle\phi_{0}\left|\theta^{(+)}(x)\right| \chi(t)\right\rangle \\
& =\left\langle\phi_{0}\left|\sum_{r=1}^{2} \int d^{3} p b_{r}(\vec{p}) \chi_{\vec{p}, r}^{(+)}(x)\right| \chi(t)\right\rangle \\
& =\frac{1}{(2 \pi \hbar)^{3 / 2}} \sum_{r=1}^{2} \int d^{3} p \sqrt{\frac{m c^{2}}{E(\vec{p})}} u^{(r)}(\vec{p}) e^{i(\vec{p} \cdot \vec{x}-E(\vec{p}) t) / \hbar}\left\langle 1_{\vec{p}, r} \mid \chi(t)\right\rangle
\end{aligned}
$$

with $\left|1_{\vec{p}, r}\right\rangle=b_{r}^{\dagger}(\vec{p})\left|\phi_{0}\right\rangle$ and with, using again (101),

$$
\begin{aligned}
& \left\langle\chi_{\mathrm{ref}} \mid \chi(t)\right\rangle=\left\langle\chi_{\mathrm{ref}}\left|U\left(t, t_{0}\right)\right| \chi_{i}\right\rangle \\
& =\left\langle\chi_{\mathrm{ref}} \mid \chi_{i}\right\rangle-\frac{i}{\hbar c} \int_{t_{0}}^{t} d^{4} x^{\prime} \chi_{\mathrm{ref}}^{\dagger}\left(x^{\prime}\right) \mathcal{V}_{G}\left(x^{\prime}\right) \chi_{i}\left(x^{\prime}\right) \\
& =\left\langle\chi_{\mathrm{ref}} \mid \chi_{i}\right\rangle-\frac{i}{\hbar} \frac{1}{(2 \pi \hbar)^{3}} \sum_{r, r^{\prime}} \int_{t_{0}}^{t} d t^{\prime} \int d^{3} x^{\prime} \int d^{3} p d^{3} p^{\prime}\left\langle\chi_{\mathrm{ref}} \mid 1_{\vec{p}, r}\right\rangle \\
& \sqrt{\frac{m c^{2}}{E(\vec{p})}} u^{(r) \dagger}(\vec{p}) e^{-i\left(\vec{p} \cdot \vec{x}^{\prime}-E(\vec{p}) t^{\prime}\right) / \hbar} \frac{1}{(2 \pi)^{3 / 2}} \int d^{3} k \widetilde{\mathcal{V}}_{G}\left(\vec{k}, \vec{p}^{\prime}, t^{\prime}\right) e^{i \vec{k} \cdot \vec{x}^{\prime}} \\
& \sqrt{\frac{m c^{2}}{E\left(\vec{p}^{\prime}\right)}} u^{\left(r^{\prime}\right)}\left(\vec{p}^{\prime}\right) e^{i\left(\vec{p}^{\prime} \cdot \vec{x}^{\prime}-E\left(\vec{p}^{\prime}\right) t^{\prime}\right) / \hbar}\left\langle 1_{\vec{p}^{\prime}, r^{\prime}} \mid \chi_{i}\right\rangle \\
& =\left\langle\chi_{\mathrm{ref}} \mid \chi_{i}\right\rangle-\frac{i}{\hbar} \sum_{r, r^{\prime}} \int_{t_{0}}^{t} d t^{\prime} \int d^{3} p \int \frac{d^{3} k}{(2 \pi)^{3 / 2}}\left\langle\chi_{\mathrm{ref}} \mid 1_{\vec{p}+\hbar \vec{k}, r}\right\rangle \\
& \sqrt{\frac{m c^{2}}{E(\vec{p}+\hbar \vec{k})}} \sqrt{\frac{m c^{2}}{E(\vec{p})}} u^{(r) \dagger}(\vec{p}+\hbar \vec{k}) \widetilde{\mathcal{V}}_{G}\left(\vec{k}, \vec{p}, t^{\prime}\right) u^{\left(r^{\prime}\right)}(\vec{p}) \\
& e^{i[E(\vec{p}+\hbar \vec{k})-E(\vec{p})] t^{\prime} / \hbar}\left\langle 1_{\vec{p}, r^{\prime}} \mid \chi\left(t^{\prime}\right)\right\rangle
\end{aligned}
$$


and similar expressions for antiparticles. In many cases $\left|\chi_{i}\right\rangle$ and $\left|\chi_{\text {ref }}\right\rangle$ can be conveniently taken as plane waves, but it is usually more interesting to consider wave packets. Replacing $\left|\chi_{\text {ref }}\right\rangle$ by $\left|1_{\vec{p}, r}\right\rangle$, we check that the momentum representation $\left\langle 1_{\vec{p}, r} \mid \chi(t)\right\rangle$ of $\chi(x)$ satisfies:

$$
\begin{aligned}
& i \hbar \partial_{t}\left\langle\phi_{0}\left|b_{r}(\vec{p})\right| \chi(t)\right\rangle \\
& \quad=\sum_{r^{\prime}} \int \frac{d^{3} k}{(2 \pi)^{3 / 2}} \sqrt{\frac{m c^{2}}{E(\vec{p})}} \sqrt{\frac{m c^{2}}{E(\vec{p}-\hbar \vec{k})}} u^{(r) \dagger}(\vec{p}) \widetilde{\mathcal{V}}_{G}(\vec{k}, \vec{p}-\hbar \vec{k}, t) u^{\left(r^{\prime}\right)}(\vec{p}-\hbar \vec{k}) e^{i[E(\vec{p})-E(\vec{p}-\hbar \vec{k})] t / \hbar}\left\langle\phi_{0}\left|b_{r^{\prime}}(\vec{p}-\hbar \vec{k})\right| \chi(t)\right\rangle .
\end{aligned}
$$

This equation is, in momentum representation, the analogous of equation (100) in configuration space and we shall give its first-order solution later. It leads to a discrete set of coupled equations for a fixed or negligible recoil momentum. We illustrate below, in the case of the scattering amplitude, how the matrix element, which appears in the second member of (113) and (114) can be evaluated.

To first order, the scattering amplitude (113) is:

$$
\begin{aligned}
&\left\langle\chi_{\mathrm{ref}} \mid \chi^{(1)}(t)\right\rangle=\left\langle\chi_{\mathrm{ref}}\left|U^{(1)}\left(t, t_{0}\right)\right| \chi_{i}\right\rangle \\
&=-\frac{i}{\hbar} \sum_{r, r^{\prime}} \int_{t_{0}}^{t} d t^{\prime} \int d^{3} p \int \frac{d^{3} k}{(2 \pi)^{3 / 2}}\left\langle\chi_{\mathrm{ref}} \mid 1_{\vec{p}+\hbar \vec{k}, r}\right\rangle \sqrt{\frac{m c^{2}}{E(\vec{p}+\hbar \vec{k})}} \sqrt{\frac{m c^{2}}{E(\vec{p})}} \\
& u^{(r) \dagger}(\vec{p}+\hbar \vec{k}) \widetilde{\mathcal{V}_{G}}\left(\vec{k}, \vec{p}, t^{\prime}\right) u^{\left(r^{\prime}\right)}(\vec{p}) e^{i[E(\vec{p}+\hbar \vec{k})-E(\vec{p})] t^{\prime} / \hbar}\left\langle 1_{\vec{p}, r^{\prime}} \mid \chi_{i}\right\rangle .
\end{aligned}
$$

The next step takes benefit from the smallness of $\hbar \vec{k} / m c$ or of $\hbar \vec{k} c / E(\vec{p})$ to expand the various quantities in this expression to first order in these parameters. The energy $E(\vec{p}+\hbar \vec{k})$ can be expanded in a Taylor series:

$$
E(\vec{p}+\hbar \vec{k})=E(\vec{p})+\frac{\hbar \vec{k} \cdot \vec{p} c^{2}}{E(\vec{p})}+\frac{(\hbar k)^{2} c^{2}}{2 E(\vec{p})}+\ldots=E(\vec{p})+\hbar \vec{k} \cdot \vec{v}+\hbar \delta+\ldots,
$$

where $\delta$ is the recoil shift. From the general transformation law of spinors in Lorentz boosts (see for example [26]):

$$
u(\vec{p})=\left[\cosh \left(\frac{\varphi}{2}\right)+\widehat{\vec{n}} \cdot \vec{\alpha} \sinh \left(\frac{\varphi}{2}\right)\right] u(0),
$$

where $\widehat{\vec{n}}$ is the unit vector along $\vec{p}$, and $\tanh \varphi=|\vec{p}| /(\gamma m c)$, we derive the following infinitesimal transformation for spinors:

$$
u(\vec{p}+\delta \vec{p})=\left\{1+\frac{\widehat{\vec{n}} \cdot \vec{\alpha}}{2} \tanh \varphi \frac{\widehat{\vec{n}} \cdot \delta \vec{p}}{p}+\frac{1}{2} \sinh \varphi\left[\frac{\vec{\alpha} \cdot \delta \vec{p}}{p}-(\widehat{\vec{n}} \cdot \vec{\alpha}) \frac{\widehat{\vec{n}} \cdot \delta \vec{p}}{p}\right]+i \sinh ^{2}\left(\frac{\varphi}{2}\right) \frac{\widehat{\vec{n}} \times \delta \vec{p}}{p} \cdot \vec{\Sigma}\right\} u(\vec{p})
$$

we have to first order in $\hbar \vec{k} / m c$ :

$$
u^{\dagger}(\vec{p}+\hbar \vec{k})=u^{\dagger}(\vec{p})\left\{1+\frac{1}{2 \gamma} \frac{\hbar \vec{k}}{m c} \cdot \vec{\alpha}-\frac{i}{2(\gamma+1)} \frac{\vec{p}}{m c} \times \frac{\hbar \vec{k}}{m c} \cdot \vec{\Sigma}\right\}
$$


where $\gamma=1 / \sqrt{1-\beta^{2}}, \vec{k}=\vec{k}_{\|}+\gamma \vec{k}_{\perp}$, the indices $\|$ and $\perp$ designate vector parts respectively parallel and perpendicular to $\vec{p}$. The term proportional to $\vec{\alpha}$ represents a boost (velocity change) and the term proportional to $\vec{\Sigma}$ a rotation (Thomas precession).

Rather than to calculate directly

$$
\sqrt{\frac{m c^{2}}{E(\vec{p}+\hbar \vec{k})}} \sqrt{\frac{m c^{2}}{E(\vec{p})}} u^{(r) \dagger}(\vec{p}+\hbar \vec{k}) \widetilde{\mathcal{V}}_{G}(\vec{k}, \vec{p}, t) u^{\left(r^{\prime}\right)}(\vec{p})
$$

it is simpler to calculate first the matrix element:

$$
\begin{aligned}
& \sqrt{\frac{m c^{2}}{E\left(\vec{p}+\frac{1}{2} \hbar \vec{k}\right)}} \sqrt{\frac{m c^{2}}{E\left(\vec{p}-\frac{1}{2} \hbar \vec{k}\right)}} u^{(r) \dagger}\left(\vec{p}+\frac{1}{2} \hbar \vec{k}\right) \widetilde{\mathcal{V}_{G}}\left(\vec{k}, \vec{p}-\frac{1}{2} \hbar \vec{k}, t\right) u^{\left(r^{\prime}\right)}\left(\vec{p}-\frac{1}{2} \hbar \vec{k}\right) \\
= & \frac{m c^{2}}{E(\vec{p})} u^{(r) \dagger}\left(\vec{p}+\frac{1}{2} \hbar \vec{k}\right)[\widetilde{A}(\vec{k}, t)-\widetilde{\vec{B}}(\vec{k}, t) \cdot \vec{p}] u^{\left(r^{\prime}\right)}\left(\vec{p}-\frac{1}{2} \hbar \vec{k}\right) \\
= & \frac{m c^{2}}{E(\vec{p})} u^{(r) \dagger}(\vec{p})[\widetilde{A}(\vec{k}, t)-\widetilde{\vec{B}}(\vec{k}, t) \cdot \vec{p}] u^{\left(r^{\prime}\right)}(\vec{p})+\frac{1}{4 \gamma} \frac{m c^{2}}{E(\vec{p})} u^{(r) \dagger}(\vec{p})\left\{\frac{\hbar \vec{k}}{m c} \cdot \vec{\alpha}, \widetilde{A}(\vec{k}, t)-\widetilde{\vec{B}}(\vec{k}, t) \cdot \vec{p}\right\}_{-} u^{\left(r^{\prime}\right)}(\vec{p}) \\
& -\frac{i}{4(\gamma+1)} \frac{m c^{2}}{E(\vec{p})} u^{(r) \dagger}(\vec{p})\left\{\frac{\vec{p}}{m c} \times \frac{\hbar \vec{k}}{m c} \cdot \vec{\Sigma}, \widetilde{A}(\vec{k}, t)-\widetilde{\vec{B}}(\vec{k}, t) \cdot \vec{p}\right\}_{+}^{\left(r^{\prime}\right)}(\vec{p}),
\end{aligned}
$$

where $\{A, B\}_{ \pm}$designate (anti)commutators. The first line gives:

$$
\begin{aligned}
& \frac{m c^{2}}{E(\vec{p})} u^{(r) \dagger}(\vec{p})[\widetilde{A}(\vec{k}, t)-\widetilde{\vec{B}}(\vec{k}, t) \cdot \vec{p}] u^{\left(r^{\prime}\right)}(\vec{p}) \\
& \quad=\left[\frac{E(\vec{p}) \widetilde{h}_{00}}{2}-c \vec{p} \cdot \widetilde{\vec{h}}+\frac{c^{2}}{2 E(\vec{p})} \vec{p} \cdot \widetilde{\vec{h}} \cdot \vec{p}\right] \delta_{r r^{\prime}}-\frac{i \hbar c}{4 \gamma}(\vec{k} \times \widetilde{\vec{h}}) \cdot w^{(r) \dagger}\left(\vec{\sigma}_{\perp}+\gamma \vec{\sigma}_{\|}\right) w^{\left(r^{\prime}\right)} \\
& \quad=\frac{c^{2}}{2 E(\vec{p})} p^{\mu} \widetilde{h}_{\mu \nu} p^{\nu} \delta_{r r^{\prime}}-\frac{i \hbar c}{4 \gamma}(\vec{k} \times \widetilde{\vec{h}}) \cdot w^{(r) \dagger} \vec{a} w^{\left(r^{\prime}\right)}
\end{aligned}
$$

where $w^{(r)}$ are Pauli two-component spinors corresponding either to helicity eigenvalues or to the two values of the $z$-component of the spin in the rest frame, and where

$$
\vec{a}=\left(\vec{\sigma}_{\perp}+\gamma \vec{\sigma}_{\|}\right)
$$


is the spatial part of the Thomas-Pauli-Lubanski 4-vector operator [30, 31]. The second line gives the term:

$$
\frac{1}{4 \gamma} \frac{m c^{2}}{E(\vec{p})} u^{(r) \dagger}(\vec{p})\left\{\frac{\hbar \vec{k}}{m c} \cdot \vec{\alpha}, \widetilde{A}(\vec{k}, t)-\widetilde{\vec{B}}(\vec{k}, t) \cdot \vec{p}\right\}_{-} u^{\left(r^{\prime}\right)}(\vec{p})=\frac{i \hbar c^{2}}{4 E(\vec{p}) \gamma}(\vec{k} \times \widetilde{\vec{h}} \cdot \vec{p}) \cdot w^{(r) \dagger} \vec{a} w^{\left(r^{\prime}\right)}
$$

The last line gives the Thomas precession terms:

$$
\begin{aligned}
& -\frac{i}{4(\gamma+1)} \frac{m c^{2}}{E(\vec{p})} u^{(r) \dagger}(\vec{p})\left\{\frac{\vec{p}}{m c} \times \frac{\hbar \vec{k}}{m c} \cdot \vec{\Sigma}, \widetilde{A}(\vec{k}, t)-\widetilde{\vec{B}}(\vec{k}, t) \cdot \vec{p}\right\}_{+} u^{\left(r^{\prime}\right)}(\vec{p}) \\
& \quad=\frac{i \hbar \widetilde{h}_{00}}{4 m(\gamma+1)}(\vec{k} \times \vec{p}) \cdot w^{(r) \dagger} \vec{a} w^{\left(r^{\prime}\right)}-\frac{i \hbar c}{2 m(\gamma+1)} \frac{\vec{p} \cdot \widetilde{\vec{h}}}{E(\vec{p})}(\vec{k} \times \vec{p}) \cdot w^{(r) \dagger} \vec{a} w^{\left(r^{\prime}\right)}+\frac{i \hbar}{4 E^{2}(\vec{p}) m(\gamma+1)}[(\vec{k} \times \vec{p}) \cdot \widetilde{\vec{h}} \cdot \vec{p}] \vec{p} \cdot w^{(r) \dagger} \vec{a} w^{\left(r^{\prime}\right)}
\end{aligned}
$$

The last line can be rewritten to yield:

$$
\begin{aligned}
& \sqrt{\frac{m c^{2}}{E\left(\vec{p}+\frac{1}{2} \hbar \vec{k}\right)}} \sqrt{\frac{m c^{2}}{E\left(\vec{p}-\frac{1}{2} \hbar \vec{k}\right)}} u^{(r) \dagger}\left(\vec{p}+\frac{1}{2} \hbar \vec{k}\right) \widetilde{\mathcal{V}_{G}}\left(\vec{k}, \vec{p}-\frac{1}{2} \hbar \vec{k}, t\right) u^{\left(r^{\prime}\right)}\left(\vec{p}-\frac{1}{2} \hbar \vec{k}\right) \\
& =\frac{c^{2}}{2 E(\vec{p})} p^{\mu} \widetilde{h}_{\mu \nu} p^{\nu} \delta_{r r^{\prime}}-\frac{i \hbar c}{4 \gamma}\left[\vec{k} \times\left(\widetilde{\vec{h}}-\widetilde{\vec{h}} \cdot \frac{\vec{p} c}{E(\vec{p})}\right)\right] \cdot w^{(r) \dagger} \vec{a} w^{\left(r^{\prime}\right)}+\frac{i \hbar}{2 m(\gamma+1)}\left[(\vec{k} \times \vec{p}) \frac{c^{2} p^{\mu} \widetilde{h}_{\mu \nu} p^{\nu}}{2 E^{2}(\vec{p})}\right] \cdot w^{(r) \dagger} \vec{a} w^{\left(r^{\prime}\right)}
\end{aligned}
$$

If we replace now $\vec{p}$ by $\vec{p}+\hbar \vec{k} / 2$ in order to calculate (115) this introduces the additional terms:

$$
\left[\frac{\hbar c^{2} \vec{k} \cdot \vec{p}}{4 E(\vec{p})}\left(\widetilde{h}_{00}-\frac{c^{2} \vec{p} \cdot \widetilde{\vec{h}} \cdot \vec{p}}{E^{2}(\vec{p})}\right)-c \frac{\hbar \vec{k}}{2} \cdot\left(\widetilde{\vec{h}}-\frac{\widetilde{\vec{h}} \cdot \vec{p} c}{E(\vec{p})}\right)\right] \delta_{r r^{\prime}}
$$

If we introduce the 4 -vector $\kappa^{\mu}$ :

$$
\begin{aligned}
\kappa^{0} c & =\vec{k} \cdot \vec{v} \\
\vec{\kappa} & =\vec{k}
\end{aligned}
$$

which corresponds to the energy-momentum 4-vector exchanged during the interaction, these terms can be rewritten:

$$
\frac{c^{2}}{2 E(\vec{p})}\left\{-p^{\mu} \widetilde{h}_{\mu \nu} p^{\nu} \frac{\hbar \kappa^{0} c}{E(\vec{p})}+\frac{\hbar \kappa^{\mu}}{2} \widetilde{h}_{\mu \nu} p^{\nu}\right\} \delta_{r r^{\prime}}
$$


Our final result is thus, for the scattering amplitude:

$$
\begin{array}{rl}
\left\langle\chi_{\mathrm{ref}}\left|U^{(1)}\left(t, t_{0}\right)\right| \chi\left(t_{0}\right)\right\rangle=-\frac{i}{\hbar} \sum_{r, r^{\prime}} \int_{t_{0}}^{t} & d t^{\prime} \int\left(d^{3} p\right) \int \frac{d^{3} k}{(2 \pi)^{3 / 2}}\left\langle\chi_{\mathrm{ref}} \mid 1_{\vec{p}+\hbar \vec{k}, r}\right\rangle\left\{\frac{c^{2}}{2 E(\vec{p})}\left(p^{\mu}+\hbar \kappa^{\mu}\right) \widetilde{h}_{\mu \nu} p^{\nu}\left(1-\frac{\hbar \kappa^{0} c}{2 E(\vec{p})}\right) \delta_{r r^{\prime}}\right. \\
& +\frac{i \hbar}{2 m(\gamma+1)}\left[(\vec{k} \times \vec{p}) \frac{c^{2} p^{\mu} \widetilde{h}_{\mu \nu} p^{\nu}}{2 E^{2}(\vec{p})}\right] \cdot w^{(r) \dagger} \vec{a} w^{\left(r^{\prime}\right)} \\
& \left.-\frac{i \hbar c}{4 \gamma}\left[\vec{k} \times\left(\widetilde{\vec{h}}-\widetilde{\vec{h}} \cdot \frac{\vec{p} c}{E(\vec{p})}\right)\right] \cdot w^{(r) \dagger} \vec{a} w^{\left(r^{\prime}\right)}\right\} e^{i[E(\vec{p}+\hbar \vec{k})-E(\vec{p})] t^{\prime} / \hbar}\left\langle 1_{\vec{p}, r^{\prime}} \mid \chi_{i}\right\rangle .
\end{array}
$$

To obtain the outgoing spinor, one can replace $\left\langle\chi_{\text {ref }}\right|$ by $\left\langle\phi_{0}\right| \theta(x)$ in the previous expression, which gives this spinor as a sum of outgoing plane-wave spinors:

$$
\begin{aligned}
& \chi(x)=\chi_{i}(x)-\frac{i}{\hbar} \frac{1}{(2 \pi \hbar)^{3 / 2}} \sum_{r, r^{\prime}} \int_{t_{0}}^{t} d t^{\prime} \int d^{3} p \int \frac{d^{3} k}{(2 \pi)^{3 / 2}} \sqrt{\frac{m c^{2}}{E(\vec{p})}} u^{(r)}(\vec{p}) e^{i[\vec{p} \cdot \vec{x}-E(\vec{p}) t / \hbar]} \\
&\left\{\frac{c^{2}}{2 E(\vec{p})} p^{\mu} \widetilde{h}_{\mu \nu}\left(p^{\nu}-\hbar \kappa^{\nu}\right)\left(1+\frac{\hbar \kappa^{0} c}{2 E(\vec{p})}\right) \delta_{r r^{\prime}}+\frac{i \hbar}{2 m(\gamma+1)}\left[(\vec{k} \times \vec{p}) \frac{c^{2} p^{\mu} \widetilde{h}_{\mu \nu} p^{\nu}}{2 E^{2}(\vec{p})}\right] \cdot w^{(r) \dagger} \vec{a} w^{\left(r^{\prime}\right)}\right. \\
&\left.\quad-\frac{i \hbar c}{4 \gamma}\left[\vec{k} \times\left(\widetilde{\vec{h}}-\tilde{\vec{h}} \cdot \frac{\vec{p} c}{E(\vec{p})}\right)\right] \cdot w^{(r) \dagger} \vec{a} w^{\left(r^{\prime}\right)}\right\} e^{i[E(\vec{p})-E(\vec{p}-\hbar \vec{k})] t^{\prime} / \hbar}\left\langle 1_{\vec{p}-\hbar \vec{k}, r^{\prime}} \mid \chi_{i}\right\rangle
\end{aligned}
$$

in which an explicit phase factor is associated with each outgoing plane wave component and which can also be obtained directly from the first-order solution of equation (114).

One can also perform the calculation presented in Appendix D, which gives this spinor in the form of Dirac matrices multiplying the initial spinor plane wave components

$$
\begin{gathered}
\chi(x)=\chi_{i}(x)-\frac{i}{\hbar} \frac{1}{(2 \pi \hbar)^{3 / 2}} \sum_{r^{\prime}} \int_{t_{0}}^{t} d t^{\prime} \int\left(d^{3} p\right) \int \frac{d^{3} k}{(2 \pi)^{3 / 2}} \frac{c^{2}}{2 E(\vec{p})}\left\{\left(p^{\mu}+\hbar \kappa^{\mu}\right) \widetilde{h}_{\mu \nu} p^{\nu}\left(1-\frac{\hbar \kappa^{0} c}{E(\vec{p})}\right)-\frac{i \hbar}{2} \kappa_{\rho} \sigma^{\rho \nu} \widetilde{h}_{\mu \nu} p^{\mu}\right\} \\
e^{i \vec{k} \cdot \vec{x}} e^{i[E(\vec{p}+\hbar \vec{k})-E(\vec{p})]\left(t^{\prime}-t\right) / \hbar \sqrt{\frac{m c^{2}}{E(\vec{p})}} u^{\left(r^{\prime}\right)}(\vec{p}) e^{i(\vec{p} \cdot \vec{x}-E(\vec{p}) t) / \hbar}\left\langle 1_{\vec{p}, r^{\prime}} \mid \chi_{i}\right\rangle} .
\end{gathered}
$$

In this formula, the integral over $\vec{p}$ can be calculated by assuming that the initial wave packet has a very narrow width in momentum space around a central value $\vec{p}_{0}$. The initial wave packet $\chi_{i}(x)$ can then be factorized. If this approximation is not sufficient an expansion of the wave packet around $\vec{p}_{0}$ can be used [22]. The $\vec{k}$ integral can be performed by turning each term linear in $\vec{k}$ into a spatial derivative and the result of Appendix $\mathrm{C}$ is recovered for the spinor in configuration space. Finally the time integral can be worked out in many cases and expresses energy conservation [22]. 
For the scattering amplitude, the comparison of equation (132) with equation (102) shows new terms directly related to the momentum exchange: a generalized Thomas precession and a generalized spin-gravitation interaction. To illustrate how this phase shift calculation is done from equation (132) we shall rewrite this equation without the terms that obviously do not contribute to the phase and use expression (116) for the energy difference, in which we neglect the recoil shift $\delta$

$$
\begin{aligned}
\delta \varphi=-\frac{1}{\hbar} \sum_{r, r^{\prime}} \int_{t_{0}}^{t} d t^{\prime} \int\left(d^{3} p\right) \alpha_{\mathrm{ref}}^{*}(\vec{p}) \alpha_{i}(\vec{p}) \beta_{r, \mathrm{ref}}^{*} \beta_{r^{\prime}, i}\left\{\frac{c^{2}}{2 E(\vec{p})} p^{\mu} h_{\mu \nu}\left(\vec{x}_{0}+\vec{v} t^{\prime}, t^{\prime}\right) p^{\nu} \delta_{r r^{\prime}}\right. \\
+\frac{\hbar}{2 m(\gamma+1)}\left[\frac{c^{2} p^{\mu} \vec{\nabla} h_{\mu \nu}\left(\vec{x}_{0}+\vec{v} t^{\prime}, t^{\prime}\right) p^{\nu}}{2 E^{2}(\vec{p})} \times \vec{p}\right] \cdot w^{(r) \dagger} \vec{a} w^{\left(r^{\prime}\right)} \\
\left.-\frac{\hbar c}{4 \gamma}\left[\vec{\nabla} \times\left(\vec{h}\left(\vec{x}_{0}+\vec{v} t^{\prime}, t^{\prime}\right)-\vec{h}\left(\vec{x}_{0}+\vec{v} t^{\prime}, t^{\prime}\right) \cdot \frac{\vec{p} c}{E(\vec{p})}\right)\right] \cdot w^{(r) \dagger} \vec{a} w^{\left(r^{\prime}\right)}\right\},
\end{aligned}
$$

where we also made explicit the centers of the wave packets and their polarization:

$$
\left\langle 1_{\vec{p}, r} \mid \chi_{i}\right\rangle=e^{-i \vec{p} \cdot \vec{x}_{0} / \hbar} \alpha_{i}(\vec{p}) \beta_{r, i},\left\langle\chi_{\mathrm{ref}} \mid 1_{\vec{p}+\hbar \vec{k}, r}\right\rangle \simeq e^{i(\vec{p}+\hbar \vec{k}) \cdot \vec{x}_{0} / \hbar} \alpha_{\mathrm{ref}}^{*}(\vec{p}) \beta_{r, \mathrm{ref}}^{*}
$$

(the assumption that the wave packet is broad enough for $\alpha_{\text {ref }}^{*}(\vec{p}+\hbar \vec{k}) \simeq \alpha_{\text {ref }}^{*}(\vec{p})$ has been made).

If we assume, for simplicity, that the reference wave packet is identical to the unperturbed wave packet: $\alpha_{\text {ref }}(\vec{p}) \beta_{r, \text { ref }} \equiv \alpha_{i}(\vec{p}) \beta_{r, i}$ and that they are very narrow in momentum space around a central value $\vec{p}$, the phase simplifies to:

$$
\begin{array}{r}
\delta \varphi=-\frac{1}{\hbar} \int_{t_{0}}^{t} d t^{\prime}\left\{\frac{c^{2}}{2 E(\vec{p})} p^{\mu} h_{\mu \nu}\left(\vec{x}_{0}+\vec{v} t^{\prime}, t^{\prime}\right) p^{\nu}+\frac{\gamma}{m(\gamma+1)}\left[\frac{c^{2} p^{\mu} \vec{\nabla} h_{\mu \nu}\left(\vec{x}_{0}+\vec{v} t^{\prime}, t^{\prime}\right) p^{\nu}}{2 E^{2}(\vec{p})} \times \vec{p}\right] \cdot \overrightarrow{\vec{s}}\right. \\
\left.-\frac{c}{2}\left[\vec{\nabla} \times\left(\vec{h}\left(\vec{x}_{0}+\vec{v} t^{\prime}, t^{\prime}\right)-\vec{h}\left(\vec{x}_{0}+\vec{v}^{\prime}, t^{\prime}\right) \cdot \frac{\vec{p} c}{E(\vec{p})}\right)\right] \cdot \overrightarrow{\vec{s}}\right\},
\end{array}
$$


where $\overline{\vec{s}}$ is the mean spin vector'

$$
\overline{\vec{s}}=\sum_{r, r^{\prime}} \beta_{r, i}^{*} \beta_{r^{\prime}, i} \hbar w^{(r) \dagger} \vec{a} w^{\left(r^{\prime}\right)} / 2 \gamma
$$

In fact the phase calculation is usually more involved since the previous formula applies only to the case of straight unperturbed trajectories. In practice however, one cannot always ignore the fact that, when calculating the phase to first-order for a given term of the Hamiltonian, the motion of the particles is affected by other terms. One example is the calculation of the gravitational shift within the atom beam splitters, in which one cannot ignore the important effects of the diffracting electromagnetic field on the trajectories of the particles [10, 38, 39, 40]. Gravitational phase shifts have to be calculated along these trajectories. Another example is the gravity field itself, which, on earth, gives parabolic trajectories for atoms. The phase shift for the other terms in equation (137) has to be calculated along these parabolas. A convenient way to achieve these calculations is to replace $\vec{x}_{0}+\vec{v} t^{\prime}$ and $\vec{v}$ in equation (137) by the classical trajectory $\left\{\vec{x}\left(t^{\prime}\right), \vec{v}\left(t^{\prime}\right)\right\}$ obtained in the $A B C D$ formalism developed in references [22, 37].

Expression (137) displays all the terms which may lead to a gravitational phase shift in a matterwave interferometer. They are summarized in Table 1 where one finds successively:

- the terms involving $h_{00}$ lead to the gravitational shift $\left(h_{00}=-2 \vec{g} \cdot \vec{r} / c^{2}\right)$, to shifts involving higher derivatives of the gravitational potential and to the analog of the Thomas precession (spin-orbit coupling corrected by the Thomas factor).

- the terms which involve $\vec{h}=\left\{h^{0 k}\right\}$, give the Sagnac effect in a rotating frame $(\vec{h}=\vec{\Omega} \times \overrightarrow{\mathbf{r}} / c)$, the spin-rotation coupling and a relativistic correction (analogous to the Thomas term for $\left.h_{00}\right)$. They describe also the Lense-Thirring effects coming from inertial frame-dragging by a massive rotating body, which is a source for $\vec{h}$.

- the other terms, which involve the tensor $\vec{h}=\left\{h^{i j}\right\}$ describe genuine General Relativity effects such as the effect of gravitational waves and de Sitter geodetic precession (which also includes the Thomas term for $\left.h_{00}\right)$.

Our expressions are valid for spins 0 and $1 / 2$ and may be conjectured to be valid for arbitrary spin if $\vec{\sigma} / 2$ is replaced by the corresponding spin operator $\vec{S}$.

The reader will find calculations of the phases corresponding to these various terms in references [3, 17, 10, 35, 41, 42, 43, 44. In these calculations, one should never forget that the external field $h_{\mu \nu}$ acts not only on the atoms but also on other components of the experiments, such as mirrors and laser beams and that, depending on the chosen gauge, additional contributions may enter in the final expression of the phase which should, of course, be gauge independent.

In present experiments on the earth gravity measurements, the relative sensitivity is $\delta g / g \simeq$ $3.10^{-9}$ after 60 seconds and the absolute accuracy $5.10^{-9}[6$, 35]. For rotations, the best sensitivity achieved up to now is $6.10^{-10} \mathrm{rad} . \mathrm{s}^{-1} \mathrm{~Hz}^{-1 / 2}$ [36] but these numbers are expected to improve rapidly in the near future, especially in space experiments, in which general relativistic effects should become detectable. An accurate measurement of the effect of gravitation and inertia on antimatter also appears as a possibility already discussed in reference [47] with a transmission-grating interferometer, although we believe that an antiatom interferometer using laser beams for the antihydrogen beam splitters (Ramsey-Bordé interferometers) would be better suited for such an experiment. The formalism introduced in this paper to deal with antiatoms should be useful to discuss such experiments, especially when coherent beams of antihydrogen will be produced either by Bose-Einstein condensation and/or by stimulated bosonic amplification.

${ }^{9}$ More generally, if we use $a_{i}(\vec{p}, r)$ instead of $\alpha_{i}(\vec{p}) \beta_{r, i}$, the mean spin vector should be written:
\[ \overrightarrow{\vec{s}}=\left\langle\chi_{i}\left|\frac{\hbar}{2} \int\left(d^{3} x\right) \theta^{\dagger}(x) \vec{\Sigma} \theta(x)\right| \chi_{i}\right\rangle=\sum_{r, r^{\prime}} \int\left(d^{3} p\right) a_{i}^{*}(\vec{p}, r) a_{i}\left(\vec{p}, r^{\prime}\right) \hbar w^{(r) \dagger} \vec{a} w^{\left(r^{\prime}\right)} / 2 \gamma \] 


\begin{tabular}{|c|c|c|}
\hline Corresponding energy term $V$ & $h_{\mu \nu}$ & Name of the effect \\
\hline \multirow{5}{*}{$\frac{1}{2} E h_{00}$} & Newtonian potential: $h_{00}=2 U / c^{2}=-2 \vec{g} \cdot \vec{x} / c^{2}$ & Gravitational red shift \\
\hline & or acceleration field $h_{00}=2 \vec{a} \cdot \vec{x} / c^{2}$ & Acceleration shift \\
\hline & Gravity gradient $\vec{g}(z) \cdot \vec{x}=-\left(g-g^{\prime} z / 2\right) z$ & \\
\hline & or curvature $R_{0 i 0 j} x^{i} x^{j}$ & \\
\hline & Fermi gauge: $h_{00}^{F}=\ddot{h}_{+}(t-z / c) \cdot\left(x^{2}-y^{2}\right) / 2+\ddot{h}_{\times}(t-z / c) x y$ & gravitational waves \\
\hline$\frac{\gamma}{2 m(\gamma+1)}\left(\vec{\nabla} h_{00} \times \vec{p}\right) \cdot \overline{\vec{s}}$ & $h_{00}=2 U / c^{2}$ gives $V=\frac{1}{m c^{2}} \frac{\gamma}{\gamma+1}[\vec{\nabla} U \times \vec{p}] \cdot \overline{\vec{s}}$ & Thomas precession \\
\hline \multirow[t]{2}{*}{$-c \vec{p} \cdot \vec{h}$} & Rotating frame: $\vec{h}=\vec{\Omega} \times \vec{x} / c$ gives $V=-\vec{\Omega} \cdot \vec{L}$ & Sagnac effect \\
\hline & $h_{0 i}$ given by the Lense-Thirring metric & Lense-Thirring (orbital) \\
\hline \multirow{2}{*}{$-(c / 2)[\vec{\nabla} \times \vec{h}] \cdot \overline{\vec{s}}$} & $\vec{h}=\vec{\Omega} \times \vec{x} / c$ gives $V=-\vec{\Omega} \cdot \overline{\vec{s}}$ & Spin-rotation interaction \\
\hline & $h_{0 i}$ given by the Lense-Thirring metric & Lense-Thirring (spin) \\
\hline$-\frac{\gamma}{m(\gamma+1)}[\vec{\nabla}(c \vec{p} \cdot \vec{h} / E) \times \vec{p}] \cdot \overline{\vec{s}}$ & & $\sim$ Thomas for rotation \\
\hline \multirow[t]{2}{*}{$c^{2} \vec{p} \cdot \vec{h} \cdot \vec{p} / 2 E$} & $\begin{array}{l}\text { Schwarzschild metric in isotropic coordinates: } \\
h_{00}=h_{11}=h_{22}=h_{33}=2 U / c^{2} \text { gives } \\
V=p^{2} U / E \text { in addition to } E U / c^{2} \text { from } h_{00}\end{array}$ & \\
\hline & $\begin{array}{l}\text { Einstein gauge: } h_{11}=-h_{22}=h_{+}(t-z / c) \\
h_{12}=h_{21}=h_{\times}(t-z / c)\end{array}$ & $\begin{array}{l}\text { Effect of gravitational } \\
\text { waves } \\
\end{array}$ \\
\hline \multirow[t]{2}{*}{$(c / 2)[\vec{\nabla} \times(\vec{h} \cdot \vec{p} c / E)] \cdot \overrightarrow{\vec{s}}$} & $\begin{array}{c}\text { Schwarzschild metric: } U=-G M / r \\
h_{00}=h_{11}=h_{22}=h_{33}=2 U / c^{2} \\
\text { gives } V=\frac{1}{m c^{2}} \frac{1}{\gamma}[\vec{\nabla} U \times \vec{p}] \cdot \overrightarrow{\vec{s}} \text { in addition } \\
\text { to } V=\frac{1}{m c^{2}} \frac{\gamma}{\gamma+1}[\vec{\nabla} U \times \vec{p}] \cdot \overrightarrow{\vec{s}} \text { from } h_{00}\end{array}$ & $\begin{array}{c}\text { de Sitter } \\
\text { or geodetic precession }\end{array}$ \\
\hline & $\begin{array}{c}\text { Einstein gauge: } \\
h_{i j}\end{array}$ & $\begin{array}{l}\text { Interaction of the spin } \\
\text { with gravitational waves }\end{array}$ \\
\hline$\frac{\gamma}{2 m(\gamma+1)}\left[\vec{\nabla}\left(c^{2} \vec{p} \cdot \overrightarrow{\vec{h}} \cdot \vec{p} / E^{2}\right) \times \vec{p}\right] \cdot \overrightarrow{\vec{s}}$ & & $\sim$ Thomas for gravitation \\
\hline
\end{tabular}

Table 1: Classification of the various energy terms entering the expression of the phase shift. The factor $\gamma$ is the time dilation factor and should not be confused with the PPN parameter $\gamma_{P P N}$ which can also be introduced (for de Sitter precession it gives the familiar factor $\left(\gamma_{P P N}+1 / 2\right)$ ). 


\subsection{Analogy with the electromagnetic interaction}

The formulas that we have derived for the transition amplitude and for the ougoing spinor strongly suggest analogies with the electromagnetic field case. To emphasize these analogies, let us introduce the following pseudo-potential 4-vector एण

$$
\widetilde{A}^{\mu}=\frac{1}{2} \widetilde{h}^{\mu \nu} p_{\nu}
$$

then the Linet-Tourrenc term, which appears also in the generalized Thomas precession is simply

$$
\frac{c^{2}}{2 E(\vec{p})} p_{\mu} \widetilde{h}^{\mu \nu} p_{\nu}=\frac{1}{\gamma} u_{\mu} \widetilde{A}^{\mu}
$$

where $u_{\mu}$ is the 4 -velocity $p_{\mu} / m$.

The corresponding field

$$
\widetilde{\Phi}_{\mu \nu}=-i\left(\kappa_{\mu} \widetilde{A}_{\nu}-\kappa_{\nu} \widetilde{A}_{\mu}\right)=(\widetilde{\boldsymbol{E}} / c, \widetilde{\boldsymbol{B}})
$$

appears in the outgoing spinor (134) through

$$
-\frac{i \hbar}{2} \kappa_{\rho} \sigma^{\rho \nu} \widetilde{h}_{\mu \nu} p^{\mu}=\frac{\hbar}{2} \sigma^{\rho \nu} \widetilde{\Phi}_{\rho \nu}
$$

and in the generalized spin-gravitation interaction term

$$
-\frac{i \hbar c}{4 \gamma}\left[\vec{k} \times\left(\widetilde{\vec{h}}-\widetilde{\vec{h}} \cdot \frac{\vec{p} c}{E(\vec{p})}\right)\right] \cdot w^{(r) \dagger} \vec{a} w^{\left(r^{\prime}\right)}=-\frac{\hbar c^{2}}{2 \gamma E(\vec{p})} w^{(r) \dagger} \vec{a} w^{\left(r^{\prime}\right)} \cdot \widetilde{\vec{B}} .
$$

This new correspondence between the gravitational interaction and the electromagnetic interaction generalizes the so-called gravitoelectric and gravitomagnetic interactions introduced by de Witt 445 and Papini 46].

\section{Appendix A: Dirac equation in curved space-time}

For a given space-time manifold, together with its metric tensor $g_{\mu \nu}$, the Lagrangian density of the Dirac field reads [14]

$$
\mathcal{L}=\frac{\hbar c}{2} \sqrt{-g} \bar{\Psi}\left[i \gamma^{\alpha} \overrightarrow{\mathcal{D}}_{\alpha}-m c / \hbar\right] \Psi+\frac{\hbar c}{2} \sqrt{-g} \bar{\Psi}\left[-i \overline{\mathcal{D}}_{\alpha} \gamma^{\alpha}-m c / \hbar\right] \Psi
$$

with

$$
\overrightarrow{\mathcal{D}}_{\alpha}=e_{\widehat{\alpha}}{ }^{\mu}\left[\vec{\partial}_{\mu}-\frac{i}{4} e_{\widehat{\beta}}^{\nu} \nabla_{\mu} e_{\widehat{\gamma} \nu} \sigma^{\beta \gamma}\right], \quad \overleftarrow{\mathcal{D}}_{\alpha}=\left[\overleftarrow{\partial}_{\mu}+\frac{i}{4} e_{\widehat{\beta}}^{\nu} \nabla_{\mu} e_{\widehat{\gamma} \nu} \sigma^{\beta \gamma}\right] e_{\widehat{\alpha}}^{\mu}
$$

In these formulas the $e_{\widehat{\alpha}}$ 's are four-vector fields constituting an orthonormal tetrad (tetrad indices have a hat), that is satisfying the relations

$$
g_{\mu \nu} e_{\widehat{\alpha}}^{\mu} e_{\widehat{\beta}}^{\nu}=\eta_{\alpha \beta},
$$

${ }^{10}$ More rigorously, one should introduce [33]

$$
\widetilde{A}^{\mu}=\frac{1}{2} \widetilde{h}^{\mu \nu}\left(p_{\nu}+\hbar \kappa_{\nu} / 2\right),
$$

which stems directly from a compact form of the interaction Hamiltonian

$$
\begin{aligned}
\mathcal{V}_{G} & =\frac{c}{4} \alpha^{\mu} h_{\mu \nu} p^{\nu}+\text { h.c. }=\frac{c}{4}\left\{\alpha^{\mu} h_{\mu \nu}, p^{\nu}\right\}_{+} \\
\text {with } p^{0} & =-\alpha^{j} p_{j}+\gamma^{0} m c \text { and } p_{j}=i \hbar \partial_{j}
\end{aligned}
$$


with $\left(\eta_{\alpha \beta}\right)=\operatorname{diag}(+1,-1,-1,-1)$. A change of tetrad is possible according to the formula

$$
e_{\widehat{\alpha}}^{\prime \mu}=\Lambda_{\alpha}^{\beta} e_{\widehat{\beta}}^{\mu}
$$

where $\Lambda$ is a matrix of the Lorentz group defined at each point of the spacetime manifold. In such a change the Lagrangian density (147) is left invariant if the Dirac field is correspondingly transformed according to the law

$$
\Psi^{\prime}=S(\Lambda)^{-1} \Psi
$$

the matrix $S(\Lambda)$ being the usual transformation matrix under a Lorentz transformation of a Dirac spinor [17]. For an infinitesimal transformation

$$
\Lambda_{\alpha \beta}=\eta_{\alpha \beta}+\varepsilon_{\alpha \beta}, \quad \varepsilon_{\alpha \beta}=-\varepsilon_{\beta \alpha},
$$

one has

$$
S(\Lambda)^{-1}=I+\frac{i}{4} \varepsilon_{\alpha \beta} \sigma^{\alpha \beta}
$$

The field equations read

$$
\left[i \gamma^{\alpha} \overrightarrow{\mathcal{D}}_{\alpha}-m c / \hbar\right] \Psi=0, \quad \bar{\Psi}\left[-i \overline{\mathcal{D}}_{\alpha} \gamma^{\alpha}-m c / \hbar\right]=0
$$

With the invariance of $\mathcal{L}$ under the phase transformations $\Psi \rightarrow \Psi e^{-i \alpha}, \bar{\Psi} \rightarrow \bar{\Psi} e^{i \alpha}$, is associated the current density

$$
j^{\mu}=\sqrt{-g} J^{\mu}, \quad J^{\mu}=c e_{\widehat{\alpha}}^{\mu} \bar{\Psi} \gamma^{\alpha} \Psi
$$

where $J^{\mu}$ is a four-vector invariant under a change of tetrad, and one has the conservation relation in either one of the two equivalent forms

$$
\partial_{\mu} j^{\mu}=0, \quad \nabla_{\mu} J^{\mu}=0
$$

\section{Appendix B: Weak-Field approximation}

It is now assumed that space-time admits a coordinate system $\left(x^{\mu}\right)$ in which the metric tensor takes the form

$$
g_{\mu \nu}=\eta_{\mu \nu}+h_{\mu \nu}, \quad\left|h_{\mu \nu}\right| \ll 1 .
$$

According to that hypothesis, the $h_{\mu \nu}$ 's will be considered as first-order quantities, and the subsequent calculations will be valid at this order. To determine the corresponding form of the Lagrangian, it suffices to construct the tetrads associated with (157). By putting $e_{\widehat{\alpha} \mu}=\eta_{\alpha \mu}+f_{\alpha \mu}$, where $f_{\alpha \mu}$ is of the first order, one obtains from (149) and (157),

$$
f_{\alpha \beta}+f_{\beta \alpha}=h_{\alpha \beta}
$$

The general solution of these equations is of the form

$$
f_{\alpha \beta}=\frac{1}{2} h_{\alpha \beta}+\epsilon_{\alpha \beta},
$$

in which the $\epsilon_{\alpha \beta}$ 's are first-order quantities only restricted by the antisymmetry condition $\epsilon_{\alpha \beta}=$ $-\epsilon_{\beta \alpha}$. One then has

$$
e_{\widehat{\alpha} \mu}=\eta_{\alpha \mu}+\frac{1}{2} h_{\alpha \mu}+\epsilon_{\alpha \mu}
$$


Introducing the free Dirac Lagrangian (2) and the associated stress-energy tensor (3), the Lagrangian density (147) calculated at the first order and corresponding to (160) reads

$$
\mathcal{L}=\mathcal{L}_{0}-\frac{1}{2} h^{\mu \nu} T_{\mu \nu}+\frac{i \hbar c}{2} \epsilon^{\mu \nu} \bar{\Psi}\left(\gamma_{\mu} \vec{\partial}_{\nu}-\overleftarrow{\partial}_{\nu} \gamma_{\mu}\right) \Psi-\frac{\hbar c}{8} \partial_{\mu} \epsilon_{\nu \rho} \bar{\Psi}\left(\gamma^{\mu} \sigma^{\nu \rho}+\sigma^{\nu \rho} \gamma^{\mu}\right) \Psi
$$

The corresponding field equations are the following

$$
\begin{aligned}
& 0= {\left[\left(1+\frac{1}{2} h\right)\left(i \gamma^{\mu} \vec{\partial}_{\mu}-m c / \hbar\right)-\frac{i}{2} h^{\mu \nu} \gamma_{\mu} \vec{\partial}_{\nu}\right.} \\
&\left.+\frac{i}{4}\left(\partial_{\mu} h-\partial_{\nu} h_{\mu}^{\nu}\right) \gamma^{\mu}+i \epsilon^{\mu \nu} \gamma_{\mu} \vec{\partial}_{\nu}-\frac{1}{4} \partial_{\mu} \epsilon_{\nu \rho} \gamma^{\mu} \sigma^{\nu \rho}\right] \Psi \\
& 0=\bar{\Psi}\left[\left(-i \gamma^{\mu} \overleftarrow{\partial}_{\mu}-m c / \hbar\right)\left(1+\frac{1}{2} h\right)+\frac{i}{2} \overleftarrow{\partial}_{\nu} \gamma_{\mu} h^{\mu \nu}\right. \\
&\left.-\frac{i}{4}\left(\partial_{\mu} h-\partial_{\nu} h_{\mu}^{\nu}\right) \gamma^{\mu}-i \overleftarrow{\partial}_{\nu} \gamma_{\mu} \epsilon^{\mu \nu}-\frac{1}{4} \partial_{\mu} \epsilon_{\nu \rho} \sigma^{\nu \rho} \gamma^{\mu}\right]
\end{aligned}
$$

The terms depending on $h_{\mu \nu}$ are identical to those appearing in (5) and (6). The same is true for the current density, which is now

$$
j^{\mu}=c \bar{\Psi}\left[\gamma^{\mu}+\frac{1}{2} h \gamma^{\mu}-\frac{1}{2} h^{\mu \nu} \gamma_{\nu}-\epsilon^{\mu \nu} \gamma_{\nu}\right] \Psi
$$

Considered independently of the context, the equations (162) and (163) are invariant under the transformations of the Poincaré group provided that the $h_{\mu \nu}$ 's and the $\epsilon_{\mu \nu}$ 's are transformed like the components of a second-rank tensor, and $\Psi$ by the corresponding transformation law of a spinor. However, the weak-field character of $h_{\mu \nu}$ is not conserved by any finite Lorentz transformation except by the rotations. Moreover, the $h_{\mu \nu}$ 's being the basic quantities, the condition that the $\epsilon_{\mu \nu}$ 's are of first order is naturally interpreted by assuming that these latter are some linear functions of the former, that is one has

$$
\epsilon_{\mu \nu}=\alpha_{\mu \nu \rho \sigma} h^{\rho \sigma} .
$$

Such a relation is not compatible with the general tensor transformation law, on account of the symmetric or antisymmetric character of $h_{\mu \nu}$ or $\epsilon_{\mu \nu}$, but can be made compatible with the rotations by a suitable choice of the coefficients $\alpha_{\mu \nu \rho \sigma}$.

The rotational invariance of $(165)$ is equivalent to the relations

$$
\alpha^{\mu^{\prime} \nu^{\prime} \rho^{\prime} \sigma^{\prime}}=R_{\mu}{ }^{\mu^{\prime}} R_{\nu} \nu^{\nu^{\prime}} R_{\rho}{ }^{\rho^{\prime}} R_{\sigma}^{\sigma^{\prime}} \alpha^{\mu \nu \rho \sigma}
$$

where $R$ can be any rotation matrix. The corresponding solutions are given by

$$
\alpha^{0 i 0 j}=\frac{1}{2} A \delta^{i j}, \quad \alpha^{0 i j k}=0, \quad \alpha^{j k 0 i}=\frac{1}{2} B \varepsilon^{i j k}, \quad \alpha^{i j k l}=0,
$$

where $A$ and $B$ are some arbitrary parameters. If, in addition, the invariance under parity is postulated, one has to take $B=0$, giving

$$
\epsilon_{0 i}=-\epsilon_{i 0}=-A h_{0 i}, \quad \epsilon_{i j}=0,
$$

then, instead of (160),

$$
e_{\widehat{0} 0}=1+\frac{1}{2} h_{00}, \quad e_{\widehat{0} i}=\left(\frac{1}{2}-A\right) h_{0 i}, \quad e_{\hat{i} 0}=\left(\frac{1}{2}+A\right) h_{0 i}, \quad e_{\widehat{i} j}=\eta_{i j}+\frac{1}{2} h_{i j} .
$$

In particular the choice $A=0$ corresponds to the tetrad

$$
e_{\widehat{\alpha} \mu}=\eta_{\alpha \mu}+\frac{1}{2} h_{\alpha \mu},
$$

\footnotetext{
${ }^{11}$ The indices of $h_{\mu \nu}$ and $\epsilon_{\mu \nu}$ are raised with the help of $\eta^{\mu \nu}$.

${ }^{12}$ As usual, the latin indices can take the values 1,2 or 3 , and $\varepsilon^{i j k}$ is the completely antisymmetric symbol.
} 
which we call the standard tetrad associated with $h_{\mu \nu}$. The corresponding Lagrangian is identical to the Lagrangian (11), therefore the equations (5) and (6) are recovered from (162) and (163) by letting $\epsilon_{\mu \nu}=0$. We will continue to designate the corresponding field by $\Psi$.

If we write explicitly the spinorial connection in this weak-field approximation

$$
-\frac{i}{4} e_{\widehat{\beta}}^{\nu} \nabla_{\mu} e_{\widehat{\gamma} \nu} \sigma^{\beta \gamma}=\frac{i}{4} \sigma^{\lambda \mu} \partial_{\lambda} h_{\mu \nu}
$$

the equation for $\Psi$ can also be written in a simple form, analogous to the electromagnetic case, and which will find an interpretation in terms of gauge fields in the flat space-time approach of the main text

$$
i \gamma^{\nu}\left(\vec{\partial}_{\nu}+\frac{i}{4} \sigma^{\lambda \mu} \partial_{\lambda} h_{\mu \nu}-\frac{1}{2} h_{\nu}{ }^{\alpha} \vec{\partial}_{\alpha}\right) \Psi-\frac{m c}{\hbar} \Psi=0
$$

Another choice of tetrad can be made in relation with the expression of the vector current. From (155) written in the weak-field case, one has in general

$$
j^{\mu} / c=\left(1+\frac{1}{2} h\right) \bar{\Psi} \gamma^{\mu} \Psi-\frac{1}{2} h^{\mu \nu} \bar{\Psi} \gamma_{\nu} \Psi-\epsilon^{\mu \nu} \bar{\Psi} \gamma_{\nu} \Psi
$$

and then

$$
j^{0} / c=\left(1+\frac{1}{2} h\right) \bar{\Psi} \gamma^{0} \Psi-\left(\frac{1}{2} h^{0 \nu}+\epsilon^{0 \nu}\right) \bar{\Psi} \gamma_{\nu} \Psi
$$

This quantity will be proportional to the usual density $\bar{\Psi} \gamma^{0} \Psi$ if one has $\epsilon^{0 i}=-\frac{1}{2} h^{0 i}$, which is obtained with the choice $A=\frac{1}{2}$. Denoting by $\Psi^{\prime}$ the corresponding field, one has ${ }^{\mathbb{3} 3}$

$$
j^{0}=c\left(1+\frac{1}{2} h_{i}^{i}\right) \overline{\Psi^{\prime}} \gamma^{0} \Psi^{\prime}
$$

It is then possible to introduce the new field $\Theta$ by [12]

$$
\Theta=\left(1+\frac{1}{2} h_{i}^{i}\right)^{\frac{1}{2}} \Psi^{\prime} \simeq\left(1+\frac{1}{4} h_{i}^{i}\right)^{\prime} \Psi^{\prime}
$$

such that

$$
j^{0}=c \bar{\Theta} \gamma^{0} \Theta
$$

as in the free-field case. The change of field $\Psi \rightarrow \Psi^{\prime}$ corresponds to a change of tetrad as defined above, so that, from (160) and (170), one has the formulas (151), (152) and (153) written with an infinitesimal parameter $\varepsilon$ equal to $-\epsilon$ that is such that $\varepsilon_{i j}=0, \varepsilon_{0 i}=-\varepsilon_{i 0}=\frac{1}{2} h_{0 i}$. This gives

$$
\Psi^{\prime}=\left(I-\frac{1}{4} h_{0 i} \gamma^{0} \gamma^{i}\right) \Psi
$$

then, from (176),

$$
\Theta=\left(I+\frac{1}{4} h-\frac{1}{4} h_{0 \mu} \gamma^{0} \gamma^{\mu}\right) \Psi .
$$

The field $\Theta$ is identical to the field introduced in Section 3.2 for the purpose of defining the quantization and the interaction picture.

\footnotetext{
${ }^{13}$ Let us note that, in the expression of the conserved charge corresponding to $(175)$, the volume element $\left(1+\frac{1}{2} h^{i}{ }_{i}\right) d^{3} x$ is, in the first-order approximation, the 3 -volume associated with the spatial metric defined from the coordinate system $\left(x^{\mu}\right)$ [20].
} 


\section{Appendix C: A stationary phase calculation}

The outgoing spinor can be calculated directly in configuration space with the help of a stationary phase formula. In the particle case, from the definition

$$
\chi(x)=\left\langle\phi_{0}\left|\theta^{(+)}(x)\right| \chi(t)\right\rangle
$$

and the integral equation

$$
|\chi(t)\rangle=\left|\chi\left(t_{0}\right)\right\rangle-\frac{i}{\hbar c} \int_{t_{0}}^{t}\left(d^{4} x^{\prime}\right) \theta^{(+) \dagger}\left(x^{\prime}\right) \mathcal{V}_{G}\left(x^{\prime}\right) \theta^{(+)}\left(x^{\prime}\right)\left|\chi\left(t^{\prime}\right)\right\rangle
$$

one can derive, to first order, the expression

$$
\chi(x)=\chi_{i}(x)-\frac{i}{\hbar c} \int_{t_{0}}^{t}\left(d^{4} x^{\prime}\right)\left\langle\phi_{0}\left|\theta^{(+)}(x) \overline{\theta^{(+)}\left(x^{\prime}\right)}\right| \phi_{0}\right\rangle \gamma^{0} \mathcal{V}_{G}\left(x^{\prime}\right)\left\langle\phi_{0}\left|\theta^{(+)}\left(x^{\prime}\right)\right| \chi_{i}\right\rangle,
$$

which, with the help of standard formulas, can be transformed into

$$
\begin{aligned}
\chi(x) & =\chi_{i}(x)-\frac{1}{\hbar c} \int_{t_{0}}^{t} d^{4} x^{\prime} S^{(+)}\left(x-x^{\prime}\right) \gamma^{0} \mathcal{V}_{G}\left(x^{\prime}\right) \chi_{i}\left(x^{\prime}\right) \\
& =\chi_{i}(x)-\frac{i}{\hbar c} \sum_{r} \int d^{3} p \chi_{\vec{p}, r}^{(+)}(x) \int_{t_{0}}^{t}\left(d^{4} x^{\prime} \overline{\chi_{\vec{p}, r}^{(+)}\left(x^{\prime}\right)} \gamma^{0} \mathcal{V}_{G}\left(x^{\prime}\right) \chi_{i}\left(x^{\prime}\right)\right.
\end{aligned}
$$

By introducing (85), one finds

$$
\chi(x)=\chi_{i}(x)-\frac{i}{\hbar c} \sum_{r} \int d^{3} p \chi_{\vec{p}, r}^{(+)}(x) i \hbar c\left\langle\chi_{\vec{p}, r}^{(+)}\left|U^{(1)}\left(t, t_{0}\right)\right| \chi_{i}\right\rangle .
$$

In this last expression the matrix element can be submitted to the same transformations as those leading from (86) to (90), yielding

$$
\begin{aligned}
\chi(x) & =\chi_{i}(x)-i \sum_{r} \int d^{3} p \chi_{\vec{p}, r}^{(+)}(x) \int_{t_{0}}^{t} d^{4} x^{\prime} \frac{i}{4} h^{\mu \nu}\left(x^{\prime}\right) \overline{\chi_{\vec{p}, r}^{(+)}\left(x^{\prime}\right)}\left(\gamma_{\mu} \overrightarrow{\partial_{\nu}^{\prime}}-\overleftarrow{\partial_{\nu}^{\prime}} \gamma_{\mu}\right) \chi_{i}\left(x^{\prime}\right) \\
& =\chi_{i}(x)-\frac{i}{4} \int_{t_{0}}^{t} d^{4} x^{\prime} h^{\mu \nu}\left(x^{\prime}\right) S^{(+)}\left(x-x^{\prime}\right)\left(\gamma_{\mu} \overrightarrow{\partial_{\nu}^{\prime}}-\overleftarrow{\partial_{\nu}^{\prime}} \gamma_{\mu}\right) \chi_{i}\left(x^{\prime}\right) .
\end{aligned}
$$

By introducing the expression

$$
S^{(+)}\left(x-x^{\prime}\right)=\left.\frac{i c}{2(2 \pi \hbar)^{3}} \int \frac{d^{3} p}{E(\vec{p})}\left(\gamma^{\mu} p_{\mu}+m c\right) e^{-i p\left(x-x^{\prime}\right) / \hbar}\right|_{p_{0}=+E(\vec{p}) / c},
$$

one finally gets for a plane wave with the momentum $\vec{p}_{0}$

$$
\begin{aligned}
\chi(x)= & \chi_{i}(x)-\frac{i c^{2}}{8 \hbar} \int_{t_{0}}^{t} d t^{\prime} \int d^{3} x^{\prime} h^{\mu \nu}\left(x^{\prime}\right) \times \\
& \times \frac{1}{(2 \pi \hbar)^{3}} \int \frac{d^{3} p}{E(\vec{p})}\left(p+p_{0}\right)_{\nu}\left(\gamma^{\rho} p_{\rho}+m c\right) e^{-i p\left(x-x^{\prime}\right) / \hbar} \gamma_{\mu} \chi_{i}\left(x^{\prime}\right) .
\end{aligned}
$$


The elementary stationary phase formula ${ }^{14}$ applied successively to the integral over $p$, then to the integral over $x^{\prime}$, yields the Linet-Tourrenc result [23], namely :

$$
\chi(x)=\left[1-\frac{i c^{2}}{2 \hbar} \frac{p_{0 \mu} p_{0 \nu}}{E\left(\overrightarrow{p_{0}}\right)} \int_{t_{0}}^{t} d t^{\prime} h^{\mu \nu}\left(\vec{x}-\vec{v}_{0}\left(t-t^{\prime}\right), t^{\prime}\right)\right] \chi_{i}(x), \quad \vec{v}_{0}=\frac{\vec{p}_{0} c^{2}}{E\left(\vec{p}_{0}\right)} .
$$

This expression is the beginning of an asymptotic expansion in powers of $\hbar$ of the form

$$
\chi(x)=\left[1-\frac{i c^{2}}{2 \hbar} \int_{t_{0}}^{t} d t^{\prime} F\left(\vec{x}-\vec{v}_{0}\left(t-t^{\prime}\right), t^{\prime}\right)\right] \chi_{i}(x)
$$

in which $F$ is defined by an expansion in integer powers of $\hbar$ whose first term corresponds to the Linet-Tourrenc formula. This expansion can be obtained from a generalization of the stationary phase formula given by Hörmander [25]. The application of this general formula to the two integrals appearing in (188) yields, after some complicated calculations, the following expression of the outgoing spinor (to be derived in the next Appendix by a simpler method)

$$
\begin{aligned}
F=\left\{\frac{p_{\mu} p_{\nu} h^{\mu \nu}}{E(\vec{p})}+\frac{i \hbar}{2 E(\vec{p})}\right. & {\left[p_{\nu} \partial_{i} h^{\mu \nu} \gamma^{i} \gamma_{\mu}+p_{\mu} \partial_{i} h^{\mu i}-p_{\mu} \frac{v^{i}}{c}\left(\partial_{i} h^{\mu 0}+\partial_{i} h^{\mu \nu} \gamma^{0} \gamma_{\nu}\right)\right.} \\
+ & \left.\left.2 \frac{p_{\mu} p_{\nu}}{E(\vec{p})} v^{i} \partial_{i} h^{\mu \nu}+\frac{c^{2}\left(t-t^{\prime}\right)}{E(\vec{p})}\left(\delta^{i j}-\frac{v^{i}}{c} \frac{v^{j}}{c}\right) \partial_{i} \partial_{j}\left(p_{\mu} p_{\nu} h^{\mu \nu}\right)\right]\right\}_{\vec{p}=\vec{p}_{0}} .
\end{aligned}
$$

\section{Appendix D: Derivation of the wave function using the momentum representation}

The method used for the amplitude in the main text can be used to derive the wave function. The spinorial wave function for one-(anti)particle states is

$$
\chi(x)=\left\langle\phi_{0}|\theta(x)| \chi(t)\right\rangle .
$$

For particles:

$$
\begin{gathered}
\left\langle\phi_{0}\left|\theta^{(+)}(x)\right| \chi(t)\right\rangle=\left\langle\phi_{0}\left|\theta^{(+)}(x)\right| \chi_{i}\right\rangle-\frac{i}{\hbar} \frac{1}{(2 \pi \hbar)^{3 / 2}} \sum_{r, r^{\prime}} \int_{t_{0}}^{t} d t^{\prime} \int d^{3} p \int \frac{d^{3} k}{(2 \pi)^{3 / 2}} \frac{m c^{2}}{E(\vec{p}+\hbar \vec{k})} u^{(r)}(\vec{p}+\hbar \vec{k}) u^{(r) \dagger}(\vec{p}+\hbar \vec{k}) \\
\widetilde{\mathcal{V}}_{G}\left(\vec{k}, \vec{p}, t^{\prime}\right) e^{i \vec{k} \cdot \vec{x}} e^{\frac{i}{\hbar}[E(\vec{p}+\hbar \vec{k})-E(\vec{p})]\left(t^{\prime}-t\right)} \sqrt{\frac{m c^{2}}{E(\vec{p})}} u^{\left(r^{\prime}\right)}(\vec{p}) e^{\frac{i}{\hbar}(\vec{p} \cdot \vec{x}-E(\vec{p}) t)}\left\langle 1_{\vec{p}, r^{\prime}} \mid \chi\left(t^{\prime}\right)\right\rangle
\end{gathered}
$$

\footnotetext{
${ }^{14}$ See, for instance $[24]$.
} 
and a similar formula for antiparticles. To first order we get

$$
\begin{array}{r}
\chi^{(1)}(x)=-\frac{i}{\hbar} \frac{1}{(2 \pi \hbar)^{3 / 2}} \sum_{r, r^{\prime}} \int_{t_{0}}^{t} d t^{\prime} \int d^{3} p \int \frac{d^{3} k}{(2 \pi)^{3 / 2}} \frac{m c^{2}}{E(\vec{p}+\hbar \vec{k})} u^{(r)}(\vec{p}+\hbar \vec{k}) u^{(r) \dagger}(\vec{p}+\hbar \vec{k}) \\
\widetilde{\mathcal{V}}_{G}\left(\vec{k}, \vec{p}, t^{\prime}\right) e^{i \vec{k} \cdot \vec{x}} e^{\frac{i}{\hbar}[E(\vec{p}+\hbar \vec{k})-E(\vec{p})]\left(t^{\prime}-t\right)} \sqrt{\frac{m c^{2}}{E(\vec{p})}} u^{\left(r^{\prime}\right)}(\vec{p}) e^{\frac{i}{\hbar}(\vec{p} \cdot \vec{x}-E(\vec{p}) t)}\left\langle 1_{\vec{p}, r^{\prime}} \mid \chi_{i}\right\rangle
\end{array}
$$

The next idea is to express the propagator which appears in this equation in order to write the outgoing spinor in the form of Dirac matrices multiplying the initial spinor plane wave components.

From

$$
\sum_{r} u^{(r)}(\vec{p}) \bar{u}^{(r)}(\vec{p})=\frac{1}{2 m c}\left[\gamma^{\mu} p_{\mu}+m c\right]
$$

one obtains

$$
\sum_{r} \frac{m c^{2}}{E(\vec{p}+\hbar \vec{k})} u^{(r)}(\vec{p}+\hbar \vec{k}) u^{(r) \dagger}(\vec{p}+\hbar \vec{k})=\frac{c}{2 E(\vec{p})}\left[\gamma^{\mu}\left(p_{\mu}+\hbar \kappa_{\mu}\right)+m c\right]\left(1-\frac{\hbar \kappa^{0} c}{E(\vec{p})}\right) \gamma^{0}
$$

from which, after some algebra, one finds the spinor

$$
\begin{gathered}
\chi^{(1)}(x)=-\frac{i}{\hbar} \frac{1}{(2 \pi \hbar)^{3 / 2}} \sum_{r^{\prime}} \int_{t_{0}}^{t} d t^{\prime} \int\left(d^{3} p\right) \int \frac{d^{3} k}{(2 \pi)^{3 / 2}} \frac{c^{2}}{2 E(\vec{p})}\left\{\left(p^{\mu}+\hbar \kappa^{\mu}\right) \widetilde{h}_{\mu \nu} p^{\nu}\left(1-\frac{\hbar \kappa^{0} c}{E(\vec{p})}\right)-\frac{i \hbar}{2} \kappa_{\rho} \sigma^{\rho \nu} \widetilde{h}_{\mu \nu} p^{\mu}\right\} \\
e^{i \vec{k} \cdot \vec{x}} e^{i[E(\vec{p}+\hbar \vec{k})-E(\vec{p})]\left(t^{\prime}-t\right) / \hbar \sqrt{\frac{m c^{2}}{E(\vec{p})}} u^{\left(r^{\prime}\right)}(\vec{p}) e^{i(\vec{p} \cdot \vec{x}-E(\vec{p}) t) / \hbar}\left\langle 1_{\vec{p}, r^{\prime}} \mid \chi_{i}\right\rangle}
\end{gathered}
$$

where the time-dependent exponential can be expanded to any desired order for recoil shift corrections:

$$
e^{i[E(\vec{p}+\hbar \vec{k})-E(\vec{p})]\left(t^{\prime}-t\right) / \hbar}=e^{i \vec{k} \cdot \vec{v}\left(t^{\prime}-t\right)}\left[1+i \hbar \frac{c^{2}\left(t^{\prime}-t\right)}{2 E(\vec{p})}\left(\vec{k}^{2}-\left(\frac{c \vec{p} \cdot \vec{k}}{E(\vec{p})}\right)^{2}\right)\right] \simeq e^{i \vec{k} \cdot \vec{v}\left(t^{\prime}-t\right)}\left[1+i \delta\left(t^{\prime}-t\right)\right] .
$$

Finally one can check that:

$$
\left\langle\chi_{\mathrm{ref}}\left|U^{(1)}\left(t, t_{0}\right)\right| \chi\left(t_{0}\right)\right\rangle=\int d^{3} x \chi_{\mathrm{ref}}^{\dagger}(x) \chi^{(1)}(x)
$$


We obtain indeed

$$
\begin{aligned}
\int d^{3} x \chi_{\mathrm{ref}}^{\dagger}(x) \chi^{(1)}(x)= & -\frac{i}{\hbar} \sum_{r, r^{\prime}} \int_{t_{0}}^{t} d t^{\prime} \int d^{3} p \int \frac{d^{3} k}{(2 \pi)^{3 / 2}} e^{\frac{i}{\hbar}[E(\vec{p}+\hbar \vec{k})-E(\vec{p})] t^{\prime}} \sqrt{\frac{m c^{2}}{E(\vec{p}+\hbar \vec{k})}} u^{(r) \dagger}(\vec{p}+\hbar \vec{k})\left\langle\chi_{\mathrm{ref}} \mid 1_{\vec{p}+\hbar \vec{k}, r}\right\rangle \\
& \frac{c^{2}}{2 E(\vec{p})}\left\{\left(p^{\mu}+\hbar \kappa^{\mu}\right) \widetilde{h}_{\mu \nu} p^{\nu}\left(1-\frac{\hbar \kappa^{0} c}{E(\vec{p})}\right)-\frac{i \hbar}{2} \kappa_{\rho} \sigma^{\rho \nu} \widetilde{h}_{\mu \nu} p^{\mu}\right\} \sqrt{\frac{m c^{2}}{E(\vec{p})}} u^{\left(r^{\prime}\right)}(\vec{p})\left\langle 1_{\vec{p}, r^{\prime}} \mid \chi_{i}\right\rangle
\end{aligned}
$$

with

$$
\begin{gathered}
\sqrt{\frac{m c^{2}}{E(\vec{p}+\hbar \vec{k})}} u^{\dagger(r)}(\vec{p}+\hbar \vec{k}) \frac{c^{2}}{2 E(\vec{p})}\left\{\left(p^{\mu}+\hbar \kappa^{\mu}\right) \widetilde{h}_{\mu \nu} p^{\nu}\left(1-\frac{\hbar \kappa^{0} c}{E(\vec{p})}\right)-i \frac{\hbar}{2} \kappa_{\rho} \sigma^{\rho \nu} \widetilde{h}_{\mu \nu} p^{\mu}\right\} \sqrt{\frac{m c^{2}}{E(\vec{p})}} u^{\left(r^{\prime}\right)}(\vec{p}) \\
=\frac{c^{2}}{2 E(\vec{p})}\left(p^{\mu}+\hbar \kappa^{\mu}\right) \widetilde{h}_{\mu \nu} p^{\nu}\left(1-\frac{\hbar \kappa^{0} c}{2 E(\vec{p})}\right) \delta_{r r^{\prime}}+\frac{i \hbar}{2 m(\gamma+1)}\left[(\vec{k} \times \vec{p}) \frac{c^{2} p^{\mu} \widetilde{h}_{\mu \nu} p^{\nu}}{2 E^{2}(\vec{p})}\right] \cdot w^{(r) \dagger} \vec{a} w^{\left(r^{\prime}\right)} \\
-\frac{i \hbar c}{4 \gamma}\left[\vec{k} \times\left(\widetilde{\vec{h}}-\widetilde{\vec{h}} \cdot \frac{\vec{p} c}{E(\vec{p})}\right)\right] \cdot w^{(r) \dagger} \vec{a} w^{\left(r^{\prime}\right)}
\end{gathered}
$$

from which equation (132) is recovered. 


\subsubsection{Acknowledgement:}

One of us (Ch.J. B.) would like to thank Dr. C. Lämmerzahl and Prof. Ph. Tourrenc for many stimulating discussions and Prof. Dr. W. Ertmer for his hospitality at the University of Hannover within the Sonderforschungsbereich 407.

\section{References}

[1] P. Laurent et al.: Cold atom clocks on earth and in space, in R. Blatt, J. Eschner, D. Leibfried and F. Schmidt-Kaler (eds.): Laser Spectroscopy, Proceedings of the 14th International Conference on Laser Spectroscopy (World Scientific, Singapore 1999), p. 41.

[2] P. Berman (ed.): Atom Interferometry (Academic Press 1997).

[3] Ch.J. Bordé: Atomic interferometry and laser spectroscopy, in: Laser Spectroscopy X (World Scientific, Singapore 1991), p. 239.

[4] U. Sterr et al.: Atom interferometry based on separated light fields, in [2] and Appl. Phys. B 54, 341 (1992).

[5] F. Riehle, Th. Kisters, A. Witte, J. Helmcke and Ch.J. Bordé: Optical Ramsey Spectroscopy in a Rotating Frame: Sagnac Effect in a Matter-Wave Interferometer, Phys. Rev. Lett. 67, 177-180 (1991).

[6] B.C. Young, M. Kasevich and S. Chu: Precision atom interferometry with light pulses, in [2] and references therein.

[7] Ch.J. Bordé: Atomic interferometry with internal state labelling, Phys. Lett. A 140, 10 (1989).

[8] Ch.J. Bordé et al.: Optical Ramsey fringes with travelling waves, Phys. Rev. A 30, 1836 (1984).

[9] Ch.J. Bordé et al.: Molecular Interferometry Experiments, Phys. Lett. A 188, 187 (1994).

[10] Ch.J. Bordé: Matter-wave interferometers: a synthetic approach, in [2].

[11] For an early treatment of a gravitational wave detector using an atom interferometric gradiometer see: Ch.J. Bordé, J. Sharma, Ph. Tourrenc and Th. Damour: Theoretical approaches to laser spectroscopy in the presence of gravitational fields, J. Physique Lettres 44, L-983 (1983).

[12] Ch.J. Bordé, A. Karasiewicz and Ph. Tourrenc: General relativistic framework for atomic interferometry, Int. J. of Mod. Phys. D 3, 157 (1994).

[13] F.W. Hehl and Wei-Tou Ni: Inertial effects of a Dirac particle, Phys. Rev. D 42, 2045 (1990).

[14] S. Weinberg: Gravitation and Cosmology (John Wiley and Sons, New York 1972).

[15] S.N. Gupta: Quantization of Einstein's gravitational field: linear approximation, Proc. Phys. Soc. A 65, 161 (1952).

[16] S.N. Gupta: Quantization of Einstein's gravitational field: general treatment, Proc. Phys. Soc. A 65, 608 (1952).

[17] J.D. Bjorken and S.D. Drell: Relativistic Quantum Mechanics, (McGraw-Hill, New York 1964).

[18] J.D. Bjorken and S.D. Drell: Relativistic Quantum Fields (McGraw-Hill, New York 1965).

[19] R.P. Feynman, F.B. Morinigo and W.G. Wagner: Feynman Lectures on Gravitation, edited by B. Hatfield (Addison-Wesley, Reading MA 1995). 
[20] L.D. Landau and E.M. Lifschitz: The Classical Theory of Fields (Addison-Wesley, Reading MA 1951).

[21] S.S. Schweber: An Introduction to Relativistic Quantum Field Theory (Harper and Row, New York 1961).

[22] Ch.J. Bordé: Quantum theory of clocks and of gravitational sensors using atom interferometry, in R. Blatt, J. Eschner, D. Leibfried and F. Schmidt-Kaler (eds.): Laser Spectroscopy, Proceedings of the 14th International Conference on Laser Spectroscopy, (World Scientific, Singapore 1999), p. 160.

[23] B. Linet et P. Tourrenc: Changement de phase dans un champ de gravitation: possibilité de détection interférentielle, Can. J. Phys. 54, 1129 (1976).

[24] M. Born and E. Wolf: Principles of Optics, third edition (Pergamon Press, Oxford 1964).

[25] L. Hörmander: The Analysis of Linear Partial Differential Operators, Vol. 1, (Springer-Verlag, Berlin 1983).

[26] V.B. Berestetskii, E.M. Lifschitz and L.P. Pitaevskii: Relativistic Quantum Theory (Pergamon Press, Oxford 1971).

[27] V. Bargmann and E.P. Wigner: Group theoretical discussion of relativistic wave equations, Proc. Nat. Acad. Sci. (USA) 34, 211 (1948).

[28] L. de Broglie: Théorie Générale des particules à spin (Méthode de fusion) (Gauthier-Villars, Paris 1943).

[29] E. Durand: 16-component theory of the spin-1 particle and its generalization to arbitrary spin, Phys. Rev. D 11, 3405 (1975).

[30] L.H. Thomas: The Kinematics of an Electron with an Axis, Phil. Mag. 3, 1 (1927).

[31] J.K. Lubanski: Sur la théorie des particules élémentaires de spin quelconque.I., Physica 9, 310 (1942).

[32] J.M. Jauch and F. Rohrlich: The Theory of Photons and Electrons (Addison-Wesley, Cambridge MA 1955).

[33] Ch.J. Bordé: A comparison of electromagnetic and weak gravitational interactions in matterwave interferometry, to be published.

[34] I.N. Bronshtein and K.A. Semendyayev: Handbook of Mathematics (Springer, Berlin 1997), p. 770 .

[35] A. Peters, K. Y. Chung and S. Chu: A measurement of gravitational acceleration by dropping atoms, Nature 400, 849 (1999).

[36] A. Landragin, T. L. Gustavson and M. A. Kasevich: Precision atomic gyroscope, in R. Blatt, J. Eschner, D. Leibfried and F. Schmidt-Kaler (eds.): Laser Spectroscopy, Proceedings of the 14th International Conference on Laser Spectroscopy (World Scientific, Singapore 1999), p. 170.

[37] Ch.J. Bordé: Propagation of Laser beams and of atomic systems, in J. Dalibard et al. (eds.): Fundamental Systems in Quantum Optics,(Elsevier 1991), p. 287.

[38] Ch.J. Bordé and C. Lämmerzahl: Atom beam interferometry as two-level particle scattering by a periodic potential, Ann. Phys. (Leipzig) 8, 83 (1999). 
[39] C. Lämmerzahl and Ch.J. Bordé: Rabi oscillations in gravitational fields: exact solution, Phys. Lett. A 203, 59 (1995).

[40] C. Lämmerzahl and Ch.J. Bordé: Atom interferometry in gravitational fields: influence of gravitation on the beam splitter, Gen. Rel. Grav., 31, 635 (1999).

[41] J. Audretsch and K.-P. Marzlin: Atom interferometry with arbitrary laser configurations: exact phase shift for potentials including inertia and gravitation, J. Phys. II (France) 4, 2073 (1994).

[42] J. Audretsch and K.-P. Marzlin: Ramsey fringes in atomic interferometry: measurability of the influence of space-time curvature, Phys. Rev. A 50, 2080 (1994).

[43] C. Lämmerzahl: Relativistic treatment of Raman light-pulse atom beam interferometer with applications in gravity theory, J. Phys. II (France) 4, 2089 (1994).

[44] P. Wolf and Ph. Tourrenc: Gravimetry using atom interferometers: some systematic effects, Phys. Lett. A 251, 241 (1999).

[45] B.S. DeWitt: Superconductors and gravitational drag, Phys. Rev. Lett. 16, 1092 (1966)

[46] G. Papini: Particle wave functions in weak gravitational fields, Nuovo Cimento 52B, 136 (1967).

[47] T.J. Phillips: Measuring the gravitational acceleration of antimatter with an antihydrogen interferometer, Hyperfine Interactions 100, 163 (1996). 